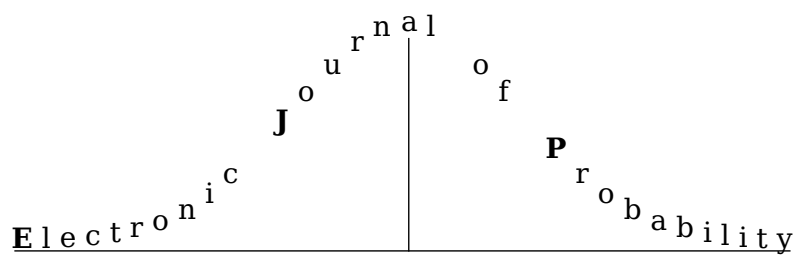

Electron. J. Probab. 25 (2020), no. 19, 1-27.

ISSN: 1083-6489 https://doi.org/10.1214/20-EJP426

\title{
Functional inequalities for weighted Gamma distribution on the space of finite measures*
}

\author{
Feng-Yu Wang ${ }^{\dagger}$
}

\begin{abstract}
Let $\mathbb{M}$ be the space of finite measures on a locally compact Polish space, and let $\mathcal{G}$ be the Gamma distribution on $\mathbb{I M}$ with intensity measure $\nu \in \mathbb{I}$. Let $\nabla^{\text {ext }}$ be the extrinsic derivative with tangent bundle $T \mathrm{IM}=\cup_{\eta \in \mathbb{M}} L^{2}(\eta)$, and let $\mathcal{A}: T \mathrm{IM} \rightarrow T \mathrm{IM}$ be measurable such that $\mathcal{A}_{\eta}$ is a positive definite linear operator on $L^{2}(\eta)$ for every $\eta \in \mathbb{M}$. Moreover, for a measurable function $V$ on $\mathbb{M}$, let $\mathrm{d} \mathcal{G}^{V}=\mathrm{e}^{V} \mathrm{~d} \mathcal{G}$. We investigate the Poincaré, weak Poincaré and super Poincaré inequalities for the Dirichlet form

$$
\mathcal{E}_{\mathcal{A}, V}(F, G):=\int_{\mathbb{M}}\left\langle\mathcal{A}_{\eta} \nabla^{e x t} F(\eta), \nabla^{e x t} G(\eta)\right\rangle_{L^{2}(\eta)} \mathrm{d} \mathcal{G}^{V}(\eta),
$$

which characterize various properties of the associated Markov semigroup. The main results are extended to the space of finite signed measures.

Keywords: extrinsic derivative; weighted Gamma distribution; Poincaré inequality; weak Poincaré inequality; super Poincaré inequality.

AMS MSC 2010: 60G57; 60G45; 60H99.

Submitted to EJP on February 9, 2019, final version accepted on January 31, 2020.
\end{abstract}

\section{Introduction}

Let $\mathrm{IM}$ be the class of finite measures on a locally compact Polish space $E$, which is again a Polish space under the weak topology. Recall that a sequence of finite measures $\eta_{n} \rightarrow \eta$ weakly if $\eta_{n}(f) \rightarrow \eta(f)$ for $f \in C_{b}(E)$, where and in what follows, for a measure $\eta$ we denote

$$
\eta(f):=\int f \mathrm{~d} \eta, \quad f \in L^{1}(\eta) .
$$

Since $M$ is locally compact, the Borel $\sigma$-algebra $\mathcal{B}(\mathbb{M})$ induced by the weak topology coincides with that induced by the vague topology. Let $\nu \in \mathbb{M}$ with $\nu(E)>0$. The Gamma distribution $\mathcal{G}$ with intensity measure $\nu$ is the unique probability measure on $\mathbb{M}$ such that for any finitely many disjoint measurable subsets $\left\{A_{1}, \cdots, A_{n}\right\}$ of $E,\left\{\eta\left(A_{i}\right)\right\}_{1 \leq i \leq n}$ are

*Supported in part by NNSFC (11771326, 11831014).

${ }^{\dagger}$ Center for Applied Mathematics, Tianjin University, Tianjin 300072, China. E-mail: wangfy@tju.edu.cn 
independent Gamma random variables with shape parameters $\left\{\nu\left(A_{i}\right)\right\}_{1 \leq i \leq n}$ and scale parameter 1; that is,

$$
\int_{\mathbb{M}} f\left(\eta\left(A_{1}\right), \cdots, \eta\left(A_{n}\right)\right) \mathcal{G}(\mathrm{d} \eta)=\int_{[0, \infty)^{n}} f\left(x_{1}, \cdots, x_{n}\right) \prod_{i=1}^{n} \gamma_{\nu\left(A_{i}\right)}\left(\mathrm{d} x_{i}\right), \quad f \in \mathcal{B}_{b}(E),
$$

where $\mathcal{B}_{b}(E)$ is the class of bounded measurable functions on $E$, for a constant $r>0$

$$
\gamma_{r}(\mathrm{~d} s):=1_{[0, \infty)}(s) \frac{s^{r-1} \mathrm{e}^{-s}}{\Gamma(r)} \mathrm{d} s, \quad \Gamma(r):=\int_{0}^{\infty} x^{r-1} \mathrm{e}^{-x} \mathrm{~d} x,
$$

and $\gamma_{0}:=\delta_{0}$ is the Dirac measure at point 0 . It is well known that $\mathcal{G}$ is concentrated on the class of finite discrete measures

$$
\mathbb{M}_{d i s}:=\left\{\sum_{i=1}^{\infty} s_{i} \delta_{x_{i}}: s_{i} \geq 0, x_{i} \in E, \sum_{i=1}^{\infty} s_{i}<\infty\right\} .
$$

Consider the weighted Gamma distribution $\mathcal{G}^{V}(\mathrm{~d} \eta):=\mathrm{e}^{V(\eta)} \mathcal{G}(\mathrm{d} \eta)$, where $V$ is a measurable function on IM. We will investigate functional inequalities for the Dirichlet form induced by $\mathcal{G}^{V}(\mathrm{~d} \eta)$ and a positive definite linear map $\mathcal{A}$ on the tangent space of the extrinsic derivative. See [7] and references therein for Dirichlet forms induced by both extrinsic and intrinsic derivatives, where the intensity measure $\nu$ is the Lebesgue measure on $\mathbb{R}^{d}$ such that the Gamma distribution $\mathcal{G}$ is concentrated on the space of infinite Radon measures on $\mathbb{R}^{d}$. In this paper, we only consider finite intensity measure $\nu$.

Definition 1.1 ([11]). A measurable real function $F$ on $\mathrm{M}$ is called extrinsically differentiable at $\eta \in \mathbb{M}$, if

$$
\nabla^{e x t} F(\eta)(x):=\left.\frac{\mathrm{d}}{\mathrm{d} s} F\left(\eta+s \delta_{x}\right)\right|_{s=0} \text { exists for all } x \in E,
$$

such that

$$
\left\|\nabla^{e x t} F(\eta)\right\|:=\left\|\nabla^{e x t} F(\eta)(\cdot)\right\|_{L^{2}(\eta)}<\infty .
$$

If $F$ is extrinsically differentiable at all $\eta \in \mathbb{M}$, we denote $F \in \mathcal{D}\left(\nabla^{e x t}\right)$ and call it extrinsically differentiable on $\mathrm{M}$.

Regarding $L^{2}(\eta)$ as the extrinsic tangent space at $\eta \in \mathbb{M}$, we define the directional derivatives by

$$
\nabla_{\phi}^{e x t} F(\eta):=\left\langle\nabla^{e x t} F(\eta), \phi\right\rangle_{L^{2}(\eta)}=\eta\left(\phi \nabla^{e x t} F(\eta)\right), \quad \phi \in L^{2}(\eta) .
$$

When $\phi$ is bounded, this coincides with the directional derivative under multiplicative actions:

$$
\nabla_{\phi}^{e x t} F(\eta)=\left.\frac{\mathrm{d}}{\mathrm{d} s} F\left(\mathrm{e}^{s \phi} \eta\right)\right|_{s=0}=\left.\frac{\mathrm{d}}{\mathrm{d} s} F((1+s \phi) \eta)\right|_{s=0}, \quad \phi \in \mathcal{B}_{b}(E),
$$

where $h \eta$ for $h \in \mathcal{B}_{b}(E)$ is a finite signed measure given by

$$
(h \eta)(A):=\eta\left(1_{A} h\right)=\int_{A} h \mathrm{~d} \eta, \quad A \in \mathcal{B}(E) .
$$

To introduce the Dirichlet form induced by the extrinsic derivative and the weighted Gamma distribution $\mathcal{G}^{V}$, we consider the class $\mathcal{F} C_{0}^{\infty}$, which consists of cylindrical functions functions of type

$$
F(\eta):=f\left(\eta\left(A_{1}\right), \cdots, \eta\left(A_{n}\right)\right), \quad n \geq 1, f \in C_{0}^{\infty}\left(\mathbb{R}^{n}\right),\left\{A_{i}\right\}_{1 \leq i \leq n} \in \mathcal{I}(E),
$$


where $\mathcal{I}(E)$ is the set of all measurable partitions of $E$. Obviously, such a function $F$ is extrinsically differentiable with

$$
\nabla^{e x t} F(\eta)=\sum_{i=1}^{n}\left(\partial_{i} f\right)\left(\eta\left(A_{1}\right), \cdots, \eta\left(A_{n}\right)\right) \cdot 1_{A_{i}} .
$$

We consider the square field

$$
\Gamma_{\mathcal{A}}(F, G):=\left\langle\mathcal{A}_{\eta} \nabla^{e x t} F(\eta), \nabla^{e x t} G(\eta)\right\rangle_{L^{2}(\eta)}=\int_{E}\left[\mathcal{A}_{\eta} \nabla^{e x t} F(\eta)\right] \cdot\left[\nabla^{e x t} G(\eta)\right] \mathrm{d} \eta,
$$

and the pre-Dirichlet form

$$
\mathcal{E}_{\mathcal{A}, V}(F, G):=\int_{\mathbb{M}} \Gamma_{\mathcal{A}}(F, G) \mathrm{d} \mathcal{G}^{V}, \quad F, G \in \mathcal{F} C_{0}^{\infty},
$$

where $\mathcal{A}$ and $V$ satisfy the following assumption.

(H) For any $\eta \in \mathbb{M}$, let $\mathcal{A}_{\eta}$ be a bounded linear operator on $L^{2}(\eta)$ such that

$$
\left\langle\mathcal{A}_{\eta} h, h\right\rangle_{L^{2}(\eta)} \geq 0, \quad h \in L^{2}(\eta),
$$

for any $A \in \mathcal{B}(E)$ and $x \in E, \mathcal{A}_{\eta} 1_{A}(x)$ is measurable in $(\eta, x) \in \mathbb{M} \times E$ and is extrinsically differentiable in $\eta$ with

$$
\sup _{\eta(E) \leq r}\left\{\left\|\mathcal{A}_{\eta}\right\|_{L^{2}(\eta)}^{2}+\left\|\nabla^{e x t}\left[\mathcal{A}_{\eta} 1_{A}\right]\right\|_{L^{2}(\eta)}\right\}<\infty, \quad r \in(0, \infty),
$$

where $\|\cdot\|_{L^{2}(\eta)}$ is the norm (or the operator norm for linear operators) in $L^{2}(\eta)$.

Moreover, $V \in \mathcal{D}\left(\nabla^{e x t}\right)$ such that

$$
\sup _{\eta(E) \leq r}\left\{|V(\eta)|+\left\|\nabla^{e x t} V(\eta)\right\|_{L^{2}(\eta)}\right\}<\infty, \quad r \in(0, \infty) .
$$

Condition (1.5) is essential for the nonnegativity of $\mathcal{E}_{\mathcal{A}, V}$, where conditions (1.6) and (1.7) ensure the boundedness of $\mathcal{A}, V$ and their extrinsic derivatives on the level sets $\{\eta(E) \leq$ $r$ f for $r>0$. These conditions are standard for establishing functional inequalities by using perturbation argument, see [14, 24] for the study of the finite-dimensional models.

We write $\mathcal{A}=\mathbf{1}$ if $\mathcal{A}_{\eta}$ is the identity map on $L^{2}(\eta)$ for every $\eta \in \mathrm{M}$. According to Theorem 3.1 below, the assumption (H) implies that $\left(\mathcal{E}_{\mathcal{A}, V}, \mathcal{F} C_{0}^{\infty}\right)$ is closable in $L^{2}\left(\mathcal{G}^{V}\right)$ and the closure $\left(\mathcal{E}_{\mathcal{A}, V}, \mathcal{D}\left(\mathcal{E}_{\mathcal{A}, V}\right)\right)$ is a symmetric Dirichlet form. If moreover

$$
\int_{\mathbb{M}}\left(1+\frac{\left\|\mathcal{A}_{\eta}\right\|_{L^{2}(\eta)}}{1+\eta(E)}\right) \mathcal{G}^{V}(\mathrm{~d} \eta)<\infty
$$

then $1 \in \mathcal{D}\left(\mathcal{E}_{\mathcal{A}, V}\right)$ with $\mathcal{E}_{\mathcal{A}, V}(1,1)=0$. Let $\left(\mathcal{L}_{\mathcal{A}, V}, \mathcal{D}\left(\mathcal{L}_{\mathcal{A}, V}\right)\right)$ be the associated generator. We aim to investigate functional inequalities for the Dirichlet form $\mathcal{E}_{\mathcal{A}, V}$ and the spectral gap of the generator $\mathcal{L}_{\mathcal{A}, V}$.

We first consider the Poincaré inequality

$$
\mathcal{G}^{V}\left(F^{2}\right) \leq \frac{1}{\lambda} \mathcal{E}_{\mathcal{A}, V}(F, F)+\mathcal{G}^{V}(F)^{2}, \quad F \in \mathcal{D}\left(\mathcal{E}_{\mathcal{A}, V}\right),
$$

where $\lambda>0$ is a constant. The spectral gap of $\mathcal{L}_{\mathcal{A}, V}$, denoted by gap $\left(\mathcal{L}_{\mathcal{A}, V}\right)$, is the largest constant $\lambda>0$ such that (1.9) holds. If (1.9) is invalid, i.e. there is no any constant $\lambda>0$ satisfying the inequality, we write $\operatorname{gap}\left(\mathcal{L}_{\mathcal{A}, V}\right)=0$ and say that $\mathcal{L}_{\mathcal{A}, V}$ does not have 
spectral gap. It is well known that (1.9) is equivalent to the exponential convergence of the associated Markov semigroup $P_{t}^{\mathcal{A}, V}$ :

$$
\left\|P_{t}^{\mathcal{A}, V} F-\mathcal{G}^{V}(F)\right\|_{L^{2}\left(\mathcal{G}^{V}\right)} \leq \mathrm{e}^{-\lambda t}\|F\|_{L^{2}\left(\mathcal{G}^{V}\right)}, \quad t \geq 0, F \in L^{2}\left(\mathcal{G}^{V}\right) .
$$

When $\operatorname{gap}\left(\mathcal{L}_{\mathcal{A}, V}\right)=0$, the following weak Poincaré inequality was introduced in [13]:

$$
\mathcal{G}^{V}\left(F^{2}\right) \leq \alpha(r) \mathcal{E}_{\mathcal{A}, V}(F, F)+r\|F\|_{\infty}^{2}, \quad F \in \mathcal{D}\left(\mathcal{E}_{\mathcal{A}, V}\right), \mathcal{G}^{V}(F)=0, r>0
$$

where $\alpha:(0, \infty) \rightarrow(0, \infty)$ corresponds to a non-exponential convergence rate of $P_{t}^{\mathcal{A}, V}$ as $t \rightarrow \infty$, see [13, Theorems 2.1 and 2.3]. In particular, (1.10) implies

$$
\left\|P_{t}^{\mathcal{A}, V}-\mathcal{G}^{V}\right\|_{L^{\infty}\left(\mathcal{G}^{V}\right) \rightarrow L^{2}\left(\mathcal{G}^{V}\right)} \leq \inf \left\{r>0: \alpha(r) \log r^{-1} \leq 2 t\right\} \downarrow 0 \text { as } t \uparrow \infty .
$$

We also consider the super Poincaré inequality

$$
\mathcal{G}^{V}\left(F^{2}\right) \leq r \mathcal{E}_{\mathcal{A}, V}(F, F)+\beta(r) \mathcal{G}^{V}(|F|)^{2}, \quad r>0, F \in \mathcal{D}\left(\mathcal{E}_{\mathcal{A}, V}\right),
$$

where $\beta:(0, \infty) \rightarrow(0, \infty)$ is a decreasing function. The existence of super Poincaré inequality is equivalent to the uniform integrability of $P_{t}^{\mathcal{A}, V}$ for $t>0$, and, when $P_{t}^{\mathcal{A}, V}$ has an asymptotic density with respect to $\mathcal{G}^{V}$, it is also equivalent to the compactness of $P_{t}^{\mathcal{A}, V}$ in $L^{2}\left(\mathcal{G}^{V}\right)$, see [24, Theorem 3.2.1] for details. According to [24, Definition 3.1.2], $P_{t}^{\mathcal{A}, V}$ is said to have an asymptotic density, if $\left\|P_{t}^{\mathcal{A}, V}-P_{n}\right\|_{L^{2}\left(\mathcal{G}^{V}\right)} \rightarrow 0$ for a sequence of bounded linear operators $\left\{P_{n}\right\}_{n \geq 1}$ having densities with respect to $\mathcal{G}^{V}$. We say that $\mathcal{E}_{\mathcal{A}, V}$ does not satisfy the super Poincaré inequality, if there is no $\beta:(0, \infty) \rightarrow(0, \infty)$ satisfying (1.11). In particular, (1.11) holds with $\beta(r)=\mathrm{e}^{c r^{-1}}$ for some constant $c>0$ if and only if the log-Sobolev inequality

$$
\mathcal{G}^{V}\left(F^{2} \log F^{2}\right) \leq C \mathcal{E}_{\mathcal{A}, V}(F, F), \quad F \in \mathcal{D}\left(\mathcal{E}_{\mathcal{A}, V}\right), \mathcal{G}^{V}\left(F^{2}\right)=1
$$

holds for some constant $C>0$. It is well known (see $[2,6])$ that (1.12) is equivalent to the hypercontractivity of $P_{t}^{\mathcal{A}, V}$ :

$$
\left\|P_{t}^{\mathcal{A}, V}\right\|_{L^{2}\left(\mathcal{G}^{V}\right) \rightarrow L^{4}\left(\mathcal{G}^{V}\right)}=1 \text { for large } t>0,
$$

as well as the exponential convergence in entropy:

$$
\mathcal{G}^{V}\left(\left(P_{t}^{\mathcal{A}, V} F\right) \log P_{t}^{\mathcal{A}, V} F\right) \leq \mathrm{e}^{-2 t / C} \mathcal{G}^{V}(F \log F), \quad t \geq 0, F \geq 0, \mathcal{G}^{V}(F)=1 .
$$

See [21, 22, 23] or [24] for more results on the super Poincaré inequalities, for instance, estimates on the semigroup $P_{t}^{\mathcal{A}, V}$ and higher order eigenvalues of the generator $\mathcal{L}_{\mathcal{A}, V}$ using the function $\beta$ in (1.11).

The remainder of the paper is organised as follows. In section 2 , we state the main results of the paper, and illustrate these results by a typical example with specific interactions. In Section 3, we establish the integration by parts formula which implies the closability of $\left(\mathcal{E}_{\mathcal{A}, V}, \mathcal{F} C_{0}^{\infty}\right)$. Then the main results are proved in Section 4 , and extended in Section 5 to the space $\mathrm{M}_{s}$ of finite signed measures.

\section{Main results and an example}

We first consider $\mathcal{E}_{\mathbf{1}, 0}$ in $L^{2}(\mathcal{G})$ whose restriction on $\mathbb{M}_{1}:=\{\mu \in \mathbb{M}: \mu(E)=1\}$ gives rise to the Dirichlet form of the Fleming-Viot process. Corresponding to results of $[16,17]$ for the Fleming-Viot process, we have the following result. See also [12, 26] for functional inequalities of different type measure-valued processes.

Theorem 2.1. Let $V=0$ and $\mathcal{A}=\mathbf{1}$. 
(1) $\operatorname{gap}\left(\mathcal{L}_{1,0}\right)=1$, i.e. $\lambda=1$ is the largest constant such that (1.9) holds for $V=0$ and $\mathcal{A}=1$.

(2) If $\operatorname{supp} \nu$ contains infinitely many points, then $\mathcal{E}_{\mathbf{1}, 0}$ does not satisfy the super Poincaré inequality.

(3) There exists a constant $c_{0}>0$ such that when $\operatorname{supp} \nu$ is a finite set, the log-Sobolev inequality

$$
\mathcal{G}\left(F^{2} \log F^{2}\right) \leq \frac{c_{0}}{1 \wedge \delta} \mathcal{E}_{\mathbf{1}, 0}(F, F), \quad F \in \mathcal{D}\left(\mathcal{E}_{\mathbf{1}, 0}\right), \mathcal{G}\left(F^{2}\right)=1
$$

holds, where $\delta:=\min \{\nu(\{x\}): x \in \operatorname{supp} \nu\}$.

To extend this result to $\mathcal{E}_{\mathcal{A}, V}$, we will adopt a split argument by making perturbations to $\mathcal{E}_{1,0}$ on bounded sets and estimating the principal eigenvalue of $\mathcal{L}_{\mathcal{A}, V}$ outside. To this end, we take

$$
\rho(\eta)=2 \sqrt{\eta(E)}, \quad \eta \in \mathbb{M}
$$

and let $\mathbf{B}_{N}=\{\eta \in \mathbb{M}: \rho(\eta) \leq N\}$ for $N>0$. Since (1.4) implies

$$
\nabla^{e x t} \rho(\eta)=\frac{1}{\sqrt{\eta(E)}}, \quad \eta \in \mathbb{M} \backslash\{0\},
$$

we have

$$
\Gamma_{\mathbf{1}}(\rho, \rho):=\eta\left(\left|\nabla^{e x t} \rho(\eta)\right|^{2}\right)=\frac{\eta(E)}{\eta(E)}=1 .
$$

According to (3.1) below, we set

$$
\mathcal{L}_{\mathcal{A}, V} \rho(\eta)=\frac{2}{\rho(\eta)}\left[(\nu-\eta)\left(\mathcal{A}_{\eta} 1\right)+\eta\left(\nabla^{e x t}\left[\mathcal{A}_{\eta} 1(\cdot)\right](\cdot)\right)+\nabla_{\mathcal{A}_{\eta} 1}^{e x t} V(\eta)\right]-\frac{4}{\rho(\eta)^{2}} \eta\left(\mathcal{A}_{\eta} 1\right)
$$

where

Let

$$
\eta\left(\nabla^{e x t}\left[\mathcal{A}_{\eta} 1(\cdot)\right](\cdot)\right):=\int_{E} \nabla^{e x t}\left[\mathcal{A}_{\eta} 1(x)\right](x) \eta(\mathrm{d} x)
$$

$$
\begin{aligned}
& \xi(r)=\sup _{\rho(\eta)=r} \mathcal{L}_{\mathcal{A}, V} \rho(\eta), \underline{a}(r)=\inf _{\rho(\eta)=r\|\phi\|_{L^{2}(\eta)}=1} \inf _{\bar{a}}\left\langle\mathcal{A}_{\eta} \phi, \phi\right\rangle_{L^{2}(\eta)}, \\
& \bar{a}(r)=\sup _{\rho(\eta)=r\|\phi\|_{L^{2}(\eta)}=1} \sup _{\mathcal{A}_{\eta}}\left\langle\mathcal{A}_{\eta} \phi\right\rangle_{L^{2}(\eta)}, \quad r>0 .
\end{aligned}
$$

Under (H), $|V(\eta)|+\left\|\mathcal{A}_{\eta}\right\|_{L^{2}(\eta)}$ is bounded on $\mathbf{B}_{r}:=\{\rho \leq r\}$ for $r \in(0, \infty)$. So, these functions are bounded on $[k, K]$ for any constants $K>k>0$. Moreover, define

$$
\sigma_{k}:=\sup _{t \geq k} \int_{t}^{\infty} \mathrm{e}^{\int_{k}^{r} \frac{\xi(s)}{\underline{a}(s)} \mathrm{d} s} \mathrm{~d} r \int_{k}^{t} \frac{1}{\underline{a}(r)} \mathrm{e}^{-\int_{k}^{r} \frac{\xi(s)}{\underline{a}(s)} \mathrm{d} s} \mathrm{~d} r, \quad k>0 .
$$

Obviously, $\sigma_{k}$ is non-increasing in $k$ and might be infinite. We will see in Theorem 2.2(1) that under certain conditions $\sigma_{k}<\infty$ implies the validity of Poincaré inequality.

We have the following extension of Theorem 2.1 to $\mathcal{E}_{\mathcal{A}, V}$. When $\operatorname{supp} \nu$ is finite the model reduces to finite-dimensional diffusions, for which one may derive super Poincaré inequalities by making perturbations to (2.1). As the present study mainly focusses on the infinite-dimensional model, we exclude this case in the following result.

Theorem 2.2. Assume (H) and (1.8). Suppose that $\underline{a}(r)^{-1}$ is locally bounded in $r \in[0, \infty)$ and

$$
\psi(s):=\int_{0}^{s}[\bar{a}(r)]^{-1 / 2} \mathrm{~d} r \uparrow \infty \text { as } s \uparrow \infty .
$$

Then the following assertions hold. 
(1) If $\lim _{k \rightarrow \infty} \sigma_{k}<\infty$ (equivalently, $\sigma_{k}<\infty$ for all $k>0$ ), then

$$
\operatorname{gap}\left(\mathcal{L}_{\mathcal{A}, V}\right) \geq \sup \left\{\frac{1}{2 \Phi\left(\psi^{-1}\left(\psi(k)+32 \sigma_{k}+1\right)\right)+32 \sigma_{k}}: k>0\right\}>0,
$$

where

$$
\Phi(N):=\left(1 \vee \frac{N^{2}}{4 \nu(E)}\right) \exp \left[\sup _{\rho \leq N} V-\inf _{\rho \leq N} V\right] \sup _{r \leq N} \underline{a}(r)^{-1}, \quad N>0 .
$$

(2) If $\operatorname{supp} \nu$ contains infinitely many points, then $\mathcal{E}_{\mathcal{A}, V}$ does not satisfy the super Poincaré inequality.

(3) The weak Poincaré inequality (1.10) holds for

$$
\alpha(r):=\inf \left\{2 \Phi(N): \mathcal{G}^{V}(\rho>N) \leq \frac{r}{1+r}\right\}, r>0 .
$$

The following result shows that the condition in Theorem 2.2(1) is sharp when $\mathcal{A}_{\eta}$ and $V(\eta)$ depend only on $\rho(\eta)$.

Corollary 2.3. Assume (H) and (1.8). Let $V(\eta)=v(\rho(\eta))$ and $\mathcal{A}_{\eta}=a(\rho(\eta)) \mathbf{1}$ for large $\rho(\eta)$ and some $a, v \in C^{1}([0, \infty))$ with $\underline{a}(r)>0$ for $r \geq 0$. Then

$$
\xi(r):=\sup _{\rho(\eta)=r} \mathcal{L}_{\mathcal{A}, V} \rho(\eta)=a(r)\left(\frac{1}{r}+v^{\prime}(r)-\frac{r}{2}\right)+\frac{r}{2} a(r), \text { for large } r>0,
$$

and $\operatorname{gap}\left(\mathcal{L}_{\mathcal{A}, V}\right)>0$ if and only if $\lim _{k \rightarrow \infty} \sigma_{k}<\infty$.

As in the proof of [14, Corollary 1.3] using [14, Theorem 1.1], it is easy to see that Theorem 2.2(2) implies the following result.

Corollary 2.4. Assume (H) and (1.8). If $\inf _{r \geq 0} \underline{a}(r)>0$ and $\lim \sup _{r \rightarrow \infty} \frac{\xi(r)}{\underline{a}(r)}<0$, then $\operatorname{gap}\left(\mathcal{L}_{\mathcal{A}, V}\right)>0$.

The above two corollaries are concerned with the validity of Poincaré inequality. On the other hand, according to Theorem 2.2(3), the weak Poincaré inequality always holds under (H), (1.8) and (2.7). We will see in the proof that the rate function $\alpha$ is derived by comparing $\mathcal{E}_{\mathcal{A}, V}$ with $\mathcal{E}_{1,0}$ on bounded sets $\mathbf{B}_{N}, N>0$. However, when these two Dirichlet forms are far away, this $\alpha$ is less sharp. As a principle, to derive a sharper weak Poincaré inequality, one should compare $\mathcal{E}_{\mathcal{A}, V}$ with a closer Dirichlet form which satisfies the Poincare inequality. In this spirit, we present below an alternative result on the weak Poincaré inequality. To state the result, we introduce the class $\mathcal{H}$ as follows.

Class $\mathcal{H}$ We denote $h \in \mathcal{H}$, if $0 \leq h \in C^{1}([0, \infty))$ with $h^{\prime}(r)>0$ for $r>0$, such that

$$
\xi_{h}(r):=\xi(r)-\frac{2}{r} h(r) \inf _{\rho(\eta)=r} \eta\left(\mathcal{A}_{\eta} 1\right), \quad r>0
$$

satisfies

$$
\sigma_{1, h}:=\sup _{t \geq 1} \int_{t}^{\infty} \mathrm{e}^{\int_{1}^{r} \frac{\xi_{h}(s)}{\underline{a}(s)} \mathrm{d} s} \mathrm{~d} r \int_{1}^{t} \frac{1}{\underline{a}(r)} \mathrm{e}^{-\int_{1}^{r} \frac{\xi_{h}(s)}{\underline{a}(s)} \mathrm{d} s} \mathrm{~d} r<\infty .
$$

It is easy to see that $\mathcal{H} \neq \emptyset$ under the conditions of Theorem 2.2 and inf $\underline{a}>0$. For any $h \in \mathcal{H}$, let $V_{h}=V-h(\rho)+c(h)$, where $c(h) \in \mathbb{R}$ is such that $\mathcal{G}^{V_{h}}$ is a probability measure on IM. By Theorem 2.2(1) with $k=1$, for any $h \in \mathcal{H}$, the Poincaré inequality

$$
\mathcal{G}^{V_{h}}\left(F^{2}\right) \leq C(h) \mathcal{E}_{\mathcal{A}, V_{h}}+\mathcal{G}^{V_{h}}(F)^{2}, \quad F \in \mathcal{D}\left(\mathcal{E}_{\mathcal{A}, V_{h}}\right)
$$

holds for

$$
C(h):=2 \Phi_{1, h}\left(\psi^{-1}\left(\psi(1)+32 \sigma_{1, h}+1\right)\right)+32 \sigma_{1, h}, \quad h \in \mathcal{H} .
$$


Theorem 2.5. Assume (H), (1.8) and (2.7). If $\mathcal{H} \neq \emptyset$, then (1.10) holds for

$$
\alpha(r):=\inf \left\{C(h) \mathrm{e}^{h(N)}: h \in \mathcal{H}, N>0 \text { with } \mathcal{G}^{V}(\rho>N) \leq \frac{r}{1+r}\right\}, r>0,
$$

where $C(h)$ is given by (2.9) and (2.11).

To conclude this section, we present below a simple example to illustrate the main results. For simplicity, we only consider $\mathcal{A}_{\eta}=1$. But by a simple comparison argument, the assertions apply also to $\mathcal{A}_{\eta}$ with $\left\langle\mathcal{A}_{\eta} \phi, \phi\right\rangle_{L^{2}(\eta)} \geq c\|\phi\|_{L^{2}(\eta)}^{2}$ for some constant $c>0$ and all $\eta \in \mathbb{M}, \phi \in L^{2}(\eta)$.

Example 2.6. Consider the following potential $V_{0}$ with interactions given by $\psi_{i} \in \mathcal{B}_{b}(E \times$ $E), i=1,2,3$ :

$$
V_{0}(\eta)=\frac{2(\eta \times \eta)\left(\psi_{1}\right)}{3 \eta(E)^{3 / 2}}+\frac{(\eta \times \eta)\left(\psi_{2}\right)}{\eta(E)}+(\eta \times \eta)\left(\psi_{3}\right)-p \log (1+\eta(E)),
$$

where $p \in \mathbb{R}$ is a constant. Let $\theta_{i}=\sup \psi_{i}, 1 \leq i \leq 3$. Assume that one of the following conditions hold:

(1) $\min \left\{\theta_{3}, \theta_{2}-1, \theta_{1} \cdot 1_{\left\{\theta_{2}=1\right\}}\right\}<0$;

(2) $\theta_{1}=\theta_{2}-1=\theta_{3}=0$ and $p>\nu(E)$.

Then $Z:=\mathcal{G}\left(\mathrm{e}^{V_{0}}\right) \leq \frac{1}{\Gamma(\nu(E))} \int_{0}^{\infty}(1+s)^{-p} s^{\nu(E)-1} \mathrm{e}^{\theta_{1} s^{1 / 2}-\left(1-\theta_{2}\right) s+\theta_{3} s^{2}} \mathrm{~d} s<\infty$, so that $\mathcal{G}^{V}$ for $V:=V_{0}-\log Z$ is a probability measure on $\mathrm{M}$, and the following assertions hold:

(a) Condition (1) implies gap $\left(\mathcal{L}_{1, V}\right)>0$;

(b) Under condition (2), let

$$
\theta=\max \left\{12 \times 1_{\left\{\left\|\psi_{3}\right\|_{\infty}>0\right\}}, 8 \times 1_{\left\{\left\|\psi_{2}-1\right\|_{\infty}>0\right\}}, 6 \times 1_{\left\{\left\|\psi_{1}\right\|_{\infty}>0\right\}}, 5\right\} .
$$

Then there exists a constant $c>0$ such that the weak Poincaré inequality (1.10) holds for

$$
\alpha(r)=c r^{-\frac{\theta}{2(p-\nu(E))}}, r>0 .
$$

Proof. Obviously, the assumptions in Theorem 2.2 hold for $V$ and $\mathcal{A}_{\eta}=1$. By definition it is easy to see that

$$
\begin{aligned}
\nabla^{e x t} V(\eta)(x)= & \frac{\eta(E) \eta\left(\psi_{1}(x, \cdot)+\psi_{1}(\cdot, x)\right)-(\eta \times \eta)\left(\psi_{1}\right)}{\eta(E)^{5 / 2}}+\eta\left(\psi_{3}(x, \cdot)+\psi_{3}(\cdot, x)\right) \\
& +\frac{\eta(E) \eta\left(\psi_{2}(x, \cdot)+\psi_{2}(\cdot, x)\right)-(\eta \times \eta)\left(\psi_{2}\right)}{\eta(E)^{2}}+\frac{p}{1+\eta(E)} .
\end{aligned}
$$

Then

$$
\begin{aligned}
\nabla_{1}^{e x t} V(\eta) & :=\eta\left(\nabla^{e x t} V(\eta)\right) \leq \theta_{1} \sqrt{\eta(E)}+\theta_{2} \eta(E)+\theta_{3} \eta(E)^{2}+\frac{p \eta(E)}{1+\eta(E)} \\
& =\frac{\theta_{1} \rho(\eta)}{2}+\frac{\theta_{2} \rho(\eta)^{2}}{4}+\frac{\theta_{3} \rho(\eta)^{4}}{8}+\frac{p \rho(\eta)^{2}}{4+\rho(\eta)^{2}}
\end{aligned}
$$

(a) If (1) holds, then $\theta_{3}<0$, or $\theta_{2}<1$, or $\theta_{3}=\theta_{2}-1=0$ and $\theta_{1}<0$. In any case, we have

$$
\limsup _{\rho(\eta) \rightarrow \infty} \mathcal{L}_{1, V} \rho(\eta)=\limsup _{\rho(\eta) \rightarrow \infty} \frac{2}{\rho(\eta)}\left(\nu(E)-\frac{\rho(\eta)^{2}}{4}+\nabla_{1}^{e x t} V(\eta)\right)<0,
$$

so that Corollary 2.4 implies gap $\left(\mathcal{L}_{1, V}\right)>0$. 
(b) Under condition (2), we prove the weak Poincaré inequality for the desired $\alpha(r)$. Since one may always take $\alpha(r) \leq 1$ in (1.10) due to $\mathcal{G}^{V}\left(F^{2}\right) \leq\|F\|_{\infty}^{2}$, it suffices to prove for small $r>0$, say $r \in(0,1]$.

It is easy to see that

$$
\mathcal{G}^{V}(\rho>N) \leq c_{0} N^{\nu(E)-p}, \quad N>0
$$

holds for some constant $c_{0}>0$. For $\varepsilon \in(0,1]$, we take $h_{\varepsilon}(s)=\varepsilon \sqrt{s}$. Since $a=1$, it is easy to check that

$$
\sigma_{1, h_{\varepsilon}} \leq c_{1} \varepsilon^{-2}
$$

for some constant $c_{1}>0$ independent of $\varepsilon \in(0,1]$. Moreover, there is a constant $c_{2}$ independent of $\varepsilon \in(0,1]$ such that

$$
\sup _{\rho \leq N} V_{h_{\varepsilon}}-\inf _{\rho \leq N} V_{h_{\varepsilon}} \leq c_{2}\left[\left\|\psi_{3}\right\|_{\infty} N^{4}+\left\|\psi_{2}-1\right\|_{\infty} N^{2}+\left\|\psi_{1}\right\|_{\infty} N+\varepsilon N+\log (1+N)\right] .
$$

Combining this with (2.11), we may find constants $c_{3}, c_{4}>0$ independent of $\varepsilon \in(0,1]$ such that

$$
C\left(h_{\varepsilon}\right) \leq c_{3}\left(\left\|\psi_{3}\right\|_{\infty} \varepsilon^{-12}+\left\|\psi_{2}-1\right\|_{\infty} \varepsilon^{-8}+\left\|\psi_{1}\right\|_{\infty} \varepsilon^{-6}+\varepsilon^{-5}\right) \leq c_{4} \varepsilon^{-\theta} .
$$

Taking this into account and applying Theorem 2.5 for

$$
N=N_{r}:=\left(\frac{2 c_{0}}{r}\right)^{\frac{1}{p-\nu(E)}}
$$

such that (2.12) implies $\mathcal{G}^{V}(\rho>N) \leq \frac{r}{2}$ as required for $r \in(0,1]$, we conclude that the weak Poincaré inequality holds for

$$
\alpha(r):=\inf _{\varepsilon \in(0,1]} C\left(h_{\varepsilon}\right) \mathrm{e}^{h_{\varepsilon}\left(N_{r}\right)} \leq \inf _{\varepsilon \in(0,1]} c_{4} \varepsilon^{-\theta} \exp \left[\varepsilon\left(2 c_{0} r^{-1}\right)^{\frac{1}{2(p-\nu(E))}}\right], \quad r \in(0,1] .
$$

Therefore, by taking $\varepsilon=1 \wedge r^{\frac{1}{2(p-\nu(E))}}$, we prove (1.10) for the desired $\alpha(r)$.

\section{The Dirichlet form}

For any $F \in \mathcal{F} C_{0}^{\infty}$, let

$$
\begin{aligned}
& \mathcal{L}_{\mathcal{A}, V} F(\eta):=\int_{E} \mathcal{A}_{\eta}\left[\nabla^{e x t} F(\eta)\right](x)(\nu-\eta)(\mathrm{d} x) \\
& \quad+\int_{E} \nabla^{e x t}\left[\mathcal{A}_{\eta}\left(\nabla^{e x t} F(\eta)\right)(x)\right](x) \eta(\mathrm{d} x)+\left\langle\nabla^{e x t} V(\eta), \mathcal{A}_{\eta}\left[\nabla^{e x t} F(\eta)\right]\right\rangle_{L^{2}(\eta)} .
\end{aligned}
$$

It is easy to see from (1.4) that when $F(\eta)=f\left(\eta\left(A_{1}\right), \cdots, \eta\left(A_{n}\right)\right)$ for some $n \geq 1, f \in$ $C_{0}^{\infty}\left(\mathbb{R}^{n}\right)$ and a measurable partition $\left\{A_{i}\right\}_{1 \leq i \leq n}$ of $E$, we have

$$
\begin{aligned}
\mathcal{L}_{\mathcal{A}, V} F(\eta)= & \left(\sum_{i=1}^{n}\left[(\nu-\eta)\left(\mathcal{A}_{\eta} 1_{A_{i}}\right)+\eta\left(\nabla^{e x t}\left[\mathcal{A}_{\eta} 1_{A_{i}}(\cdot)\right](\cdot)\right)+\nabla_{\mathcal{A}_{\eta} 1_{A_{i}}}^{e x t} V(\eta)\right] \partial_{i} f\right. \\
& \left.+\sum_{i, j=1}^{n} \eta\left(1_{A_{i}} \mathcal{A}_{\eta} 1_{A_{j}}\right)\left(\partial_{i} \partial_{j} f\right)\right)\left(\eta\left(A_{1}\right), \cdots, \eta\left(A_{n}\right)\right) .
\end{aligned}
$$

Theorem 3.1. Assume (H). Then

$$
\mathcal{E}_{\mathcal{A}, V}(F, G)=-\int_{\mathbb{M}}\left(G \mathcal{L}_{\mathcal{A}, V} F\right) \mathrm{d} \mathcal{G}^{V}, \quad F, G \in \mathcal{F} C_{0}^{\infty} .
$$

Consequently, $\left(\mathcal{E}_{\mathcal{A}, V}, \mathcal{F} C_{0}^{\infty}\right)$ is closable in $L^{2}\left(\mathrm{M}, \mathcal{G}^{V}\right)$ whose closure $\left(\mathcal{E}_{\mathcal{A}, V}, \mathcal{D}\left(\mathcal{E}_{\mathcal{A}, V}\right)\right)$ is a symmetric Dirichlet form with generator $\left(\mathcal{L}_{\mathcal{A}, V}, \mathcal{D}\left(L_{\mathcal{A}, V}\right)\right)$ being the Friedrichs extension of $\left(\mathcal{L}_{\mathcal{A}, V}, \mathcal{F} C_{0}^{\infty}\right)$. If moreover (1.8) holds, then $1 \in \mathcal{D}\left(\mathcal{E}_{\mathcal{A}, V}\right)$ and $\mathcal{E}_{\mathcal{A}, V}(1,1)=0$. 
To prove this result, we introduce the divergence operator corresponding to $\nabla^{e x t}$. To this end, we formulate the Gamma distribution $\mathcal{G}$ by using the Poisson measure $\pi_{\hat{\nu}}$ with intensity $\hat{\nu}(\mathrm{d} x, \mathrm{~d} s):=s^{-1} \mathrm{e}^{-s} \nu(d x) \mathrm{d} s$ on $\hat{E}:=E \times(0, \infty)$. Recall that $\pi_{\hat{\nu}}$ is the unique probability measure on the configuration space

$$
\boldsymbol{\Gamma}(\hat{E}):=\left\{\gamma=\sum_{i=1}^{\infty} \delta_{\left(x_{i}, s_{i}\right)}: \gamma(K)<\infty \text { for compact } K \subset \hat{E},\left(x_{i}, s_{i}\right) \in \hat{E}\right\}
$$

such that for any disjoint relatively compact subsets $\left\{\hat{A}_{i}\right\}_{1 \leq i \leq n}$ of $\hat{E},\left\{\gamma \mapsto \gamma\left(\hat{A}_{i}\right)\right\}_{1 \leq i \leq n}$ are independent random Poisson random variables with parameters $\left\{\hat{\nu}\left(\hat{A}_{i}\right)\right\}_{1 \leq i \leq n}$. Since $S(\gamma):=\sum_{i=1}^{\infty} s_{i}$ for $\gamma=\sum_{i=1}^{\infty} s_{i} \delta_{x_{i}} \in \boldsymbol{\Gamma}(\hat{E})$ satisfies

$$
\int_{\Gamma(\hat{E})} S(\gamma) \pi_{\hat{\nu}}(\mathrm{d} \gamma)=\int_{\hat{E}} s \hat{\nu}(\mathrm{d} x, \mathrm{~d} s)=\nu(E)<\infty,
$$

the measure $\pi_{\hat{\nu}}$ is concentrated on the $S$-finite configuration space

$$
\boldsymbol{\Gamma}_{f}(\hat{E}):=\left\{\gamma=\sum_{i=1}^{\infty} \delta_{\left(x_{i}, s_{i}\right)} \in \boldsymbol{\Gamma}(\hat{E}): S(\gamma):=\sum_{i=1}^{\infty} s_{i}<\infty\right\} .
$$

Lemma 3.2. The map $\Phi: \boldsymbol{\Gamma}_{f}(\hat{E}) \ni \gamma=\sum_{i=1}^{\infty} s_{i} \delta_{x_{i}} \mapsto \sum_{i=1}^{\infty} s_{i} \delta_{x_{i}} \in \mathbb{M}$ is measurable with

$$
\mathcal{G}=\pi_{\hat{\nu}} \circ \Phi^{-1} \text {. }
$$

Moreover,

$$
\begin{aligned}
& \int_{\mathbb{M}} \mathcal{G}(\mathrm{d} \eta) \int_{E} F(\eta, x) \eta(\mathrm{d} x) \\
& =\int_{\mathbb{M}} \mathcal{G}(\mathrm{d} \eta) \int_{\hat{E}} \mathrm{e}^{-s} F\left(\eta+s \delta_{x}, x\right) \nu(\mathrm{d} x) \mathrm{d} s, \quad F \in L^{1}(\mathbb{M} \times E, \mathcal{G}(\mathrm{d} \eta) \eta(\mathrm{d} x)) .
\end{aligned}
$$

Proof. Formula (3.3) was proved in [8, Theorem 6.2] for $E=\mathbb{R}^{d}$ and $\nu(\mathrm{d} x)=\theta \mathrm{d} x$ (which is an infinite measure) with $\theta>0$, by identifying the Laplace transforms of $\mathcal{G}$ and $\pi_{\hat{\nu}} \circ \Phi^{-1}$. Below we explain that the same argument works to the present setting.

Firstly, the Laplace transform of $\mathcal{G}$ is

$$
\int_{\mathbb{M}} \mathrm{e}^{-\eta(h)} \mathcal{G}(\mathrm{d} \eta)=\mathrm{e}^{-\nu(\log (1+h))}, \quad h \in \mathcal{B}^{+}(E),
$$

where $\mathcal{B}^{+}(E)$ is the class of nonnegative measurable functions on $E$. This was given by $[18,(7)]$ when $\nu$ is atomless. In general, we decompose $\nu$ into $\nu=\nu_{0}+\sum_{i=1}^{\infty} c_{i} \delta_{x_{i}}$, where $\nu_{0}$ is an atomless finite measure on $E, x_{i} \in E$ with $x_{i} \neq x_{j}$ for $i \neq j$, and $c_{i} \geq 0$ with $\sum_{i=1}^{\infty} c_{i}<\infty$. Let $E_{0}=E \backslash\left\{x_{i}: i \geq 1\right\}$. By the definition of Gamma distribution,

$$
\eta\left(h \cdot 1_{E_{0}}\right), \quad \eta\left(h \cdot 1_{\left\{x_{i}\right\}}\right), \quad i \geq 1
$$

are independent under $\mathcal{G}$, the distribution of $\eta\left(h \cdot 1_{E_{0}}\right)$ under $\mathcal{G}$ coincides with that under $\mathcal{G}_{0}$ (the Gamma distribution with intensity measure $\nu_{0}$ ), and the distribution of $\eta\left(\left\{x_{i}\right\}\right)$ under $\mathcal{G}$ coincides with the one-dimensional Gamma distribution $\gamma_{c_{i}}$ with shape parameter $c_{i}$. So, applying (3.5) for $\nu_{0}$ replacing $\nu$ due to [18, (7)], and using the Laplace transform for Gamma distributions on $\mathbb{R}_{+}$, we derive

$$
\begin{aligned}
& \int_{\mathbb{M}} \mathrm{e}^{-\eta(h)} \mathcal{G}(\mathrm{d} \eta)=\left(\int_{\mathbb{M}} \mathrm{e}^{-\eta\left(h \cdot 1_{E_{0}}\right)} \mathcal{G}(\mathrm{d} \eta)\right) \cdot \prod_{i=1}^{\infty} \int_{\mathbb{M}} \mathrm{e}^{-h\left(x_{i}\right) \eta\left(\left\{x_{i}\right\}\right)} \mathcal{G}(\mathrm{d} \eta) \\
& =\mathrm{e}^{-\nu_{0}(\log (1+h))} \cdot \prod_{i=1}^{\infty} \mathrm{e}^{-c_{i} \log \left(1+h\left(x_{i}\right)\right)}=\mathrm{e}^{-\nu(\log (1+h))}
\end{aligned}
$$


Therefore, (3.5) holds.

On the other hand, the Laplace transform for $\pi_{\hat{\nu}}$ (see for instance [1]) is

$$
\int_{\Gamma_{p f}(\hat{E})} \mathrm{e}^{-\gamma(\hat{h})} \pi_{\hat{\nu}}(\mathrm{d} \gamma)=\exp \left[-\hat{\nu}\left(1-\mathrm{e}^{-\hat{h}}\right)\right], \quad \hat{h} \in \mathcal{B}^{+}(\hat{E}) .
$$

By letting $\hat{h}(x, s)=\operatorname{sh}(x)$ for $(x, s) \in \hat{E}$, we arrive at

$$
\begin{aligned}
& \left.\int_{\mathbb{M}} \mathrm{e}^{-\eta(h)}\left(\pi_{\hat{\nu}} \circ \Phi^{-1}\right)(\mathrm{d} \eta)=\int_{\Gamma_{p f}(\hat{E})} \mathrm{e}^{-\gamma(\hat{h})} \pi_{\hat{\nu}}\right)(\mathrm{d} \gamma) \\
& =\exp \left[-\hat{\nu}\left(1-\mathrm{e}^{-\hat{h}}\right)\right]=\mathrm{e}^{-\nu(\log (1+h))}, \quad h \in \mathcal{B}^{+}(E) .
\end{aligned}
$$

Combining this with (3.5) we prove (3.3).

Finally, (3.4) follows from (3.3) and the Mecke formula [10, Satz 3.1] for Poisson measures.

To establish the integration by parts formula for $\nabla_{\phi}^{e x t} F$, we introduce the divergence operator $\operatorname{div}^{e x t}$ as follows.

Let $\phi: \mathbb{M} \times E \rightarrow \mathbb{R}$ be measurable. If for any $x \in E, \phi(\cdot, x) \in \mathcal{D}\left(\nabla^{e x t}\right)$ such that

$$
(\mathcal{G} \times \nu)(|\phi|)+\int_{\mathbb{M}} \eta\left(|\phi(\eta, \cdot)|+\left|\nabla^{e x t} \phi(\eta, \cdot)(\cdot)\right|\right) \mathcal{G}(\mathrm{d} \eta)<\infty,
$$

where $\eta(\cdot)$ stands for the integral with respect to $\eta$ as in (1.1), then we write $\phi \in \mathcal{D}$ (div ${ }^{e x t}$ ) and denote

$$
\operatorname{div}^{e x t}(\phi)(\eta)=(\eta-\nu)(\phi(\eta, \cdot))-\eta\left(\nabla^{e x t} \phi(\eta, \cdot)(\cdot)\right) .
$$

When $\phi(\eta, x)=\phi(x)$ does not depend on $\eta$, the following integration by parts formula follows from [9, Theorem 14]. We include below a complete proof for the $\eta$-dependent $\phi$.

Lemma 3.3. Let $\phi \in \mathcal{D}\left(\operatorname{div}^{e x t}\right)$. Then

$$
\int_{\mathbb{M}}\left(\nabla_{\phi}^{e x t} F\right) \mathrm{d} \mathcal{G}=\int_{\mathbb{M}}\left[F \operatorname{div}^{e x t}(\phi)\right] \mathrm{d} \mathcal{G}, \quad F \in \mathcal{F} C_{0}^{\infty} .
$$

Proof. By (3.4) and the Dominated Convergence Theorem, we obtain

$$
\begin{aligned}
& \int_{\mathbb{M}}\left(\nabla_{\phi}^{e x t} F\right) \mathrm{d} \mathcal{G}=\int_{\mathbb{M} \times E}\left(\lim _{\varepsilon \downarrow 0} \frac{F\left(\eta+\varepsilon \delta_{x}\right)-F(\eta)}{\varepsilon}\right) \phi(\eta, x) \eta(\mathrm{d} x) \mathcal{G}(\mathrm{d} \eta) \\
& =\int_{\mathbb{M}} \mathcal{G}(\mathrm{d} \eta) \lim _{\varepsilon \downarrow 0} \int_{\hat{E}} \frac{1}{\varepsilon} \mathrm{e}^{-s}\left[F\left(\eta+(s+\varepsilon) \delta_{x}\right)-F\left(\eta+s \delta_{x}\right)\right] \phi\left(\eta+s \delta_{x}, x\right) \nu(\mathrm{d} x) \mathrm{d} s \\
& =\int_{\mathbb{M}} \mathcal{G}(\mathrm{d} \eta) \int_{\hat{E}} \mathrm{e}^{-s}\left[\partial_{s} F\left(\eta+s \delta_{x}, x\right)\right] \phi\left(\eta+s \delta_{x}, x\right) \nu(\mathrm{d} x) \mathrm{d} s \\
& =\int_{\mathbb{M}} \mathcal{G}(\mathrm{d} \eta) \int_{\hat{E}}\left(\partial_{s}\left[\mathrm{e}^{-s} F\left(\eta+s \delta_{x}\right) \phi\left(\eta+s \delta_{x}, x\right)\right]-F\left(\eta+s \delta_{x}\right) \partial_{s}\left[\mathrm{e}^{-s} \phi\left(\eta+s \delta_{x}, x\right)\right]\right) \nu(\mathrm{d} x) \mathrm{d} s
\end{aligned}
$$

Noting that $F \in \mathcal{F} C_{0}^{\infty}$ implies $F\left(\eta+s \delta_{x}\right)=0$ for large $s$, we have

$$
\int_{0}^{\infty} \partial_{s}\left[\mathrm{e}^{-s} F\left(\eta+s \delta_{x}\right) \phi\left(\eta+s \delta_{x}, x\right)\right] \mathrm{d} s=-F(\eta) \phi(\eta, x) .
$$


Hence, by using (3.4) again,

$$
\begin{aligned}
& \int_{\mathbb{M}}\left(\nabla_{\phi}^{e x t} F\right) \mathrm{d} \mathcal{G}+\int_{\mathbb{M}} F(\eta) \nu(\phi(\eta, \cdot)) \mathcal{G}(\mathrm{d} \eta) \\
& =-\int_{\mathbb{M}} \mathcal{G}(\mathrm{d} \eta) \int_{\hat{E}} F\left(\eta+s \delta_{x}\right) \mathrm{e}^{-s}\left[\partial_{s} \phi\left(\eta+s \delta_{x}, x\right)-\phi\left(\eta+s \delta_{x}, x\right)\right] \nu(\mathrm{d} x) \mathrm{d} s \\
& =\int_{\mathbb{M}} \mathcal{G}(\mathrm{d} \eta) \int_{\hat{E}}\left[\phi\left(\eta+s \delta_{x}, x\right)-\nabla^{e x t} \phi(\cdot, x)\left(\eta+s \delta_{x}\right)(x)\right] \mathrm{e}^{-s} F\left(\eta+s \delta_{x}\right) \nu(\mathrm{d} x) \mathrm{d} s \\
& =\int_{\mathbb{M}} F(\eta) \mathcal{G}(\mathrm{d} \eta) \int_{E}\left[\phi(\eta, x)-\nabla^{e x t} \phi(\eta, x)(x)\right] \eta(\mathrm{d} x)
\end{aligned}
$$

Therefore, (3.7) holds.

Proof of Theorem 3.1. We first prove (3.2), which implies the closability of $\left(\mathcal{E}_{\mathcal{A}, V}, \mathcal{F} C_{0}^{\infty}\right)$ and that the closure is a symmetric Dirichlet form in $L^{2}\left(\mathcal{G}^{V}\right)$, see [4]. By the definition of $\mathcal{E}_{\mathcal{A}, V}$ and Lemma 3.3, for any $F, G \in \mathcal{F} C_{0}^{\infty}$ we have

$$
\begin{aligned}
& \mathcal{E}_{\mathcal{A}, V}(F, G)=\int_{\mathbb{M}} \Gamma_{\mathcal{A}}(F, G) \mathrm{d} \mathcal{G}^{V}=\int_{\mathbb{M}}\left(\nabla_{\mathrm{e}^{V}(\eta) \mathcal{A}_{\eta} \nabla^{e x t} F(\eta)}^{e x t}(\eta) \mathcal{G}(\mathrm{d} \eta)\right. \\
& =\int_{\mathbb{M}} G(\eta) \operatorname{div}^{e x t}\left(\mathrm{e}^{V(\eta)} \mathcal{A}_{\eta}\left[\nabla^{e x t} F(\eta)\right](\cdot)\right) \mathcal{G}(\mathrm{d} \eta)
\end{aligned}
$$

Therefore, by (3.6), (3.2) holds for

$$
\begin{aligned}
\mathcal{L}_{\mathcal{A}, V} F(\eta):= & -\mathrm{e}^{-V(\eta)} \operatorname{div}^{e x t}\left(\mathrm{e}^{V(\eta)} \mathcal{A}_{\eta}\left[\nabla^{e x t} F(\eta)\right](\cdot)\right) \\
= & \int_{E}\left(\left[\nabla^{e x t} V(\eta)(x)\right] \mathcal{A}_{\eta}\left[\nabla^{e x t} F(\eta)\right](x)+\nabla^{e x t}\left(\mathcal{A}_{\eta}\left[\nabla^{e x t} F(\eta)\right](x)\right)(x)\right) \eta(\mathrm{d} x) \\
& +\int_{E} \mathcal{A}_{\eta}\left[\nabla^{e x t} F(\eta)\right](x)(\nu-\eta)(\mathrm{d} x) .
\end{aligned}
$$

Next, assume that (1.8) holds. It remains to find a sequence $\left\{F_{n}\right\}_{n \geq 1} \subset \mathcal{D}\left(\mathcal{E}_{\mathcal{A}, V}\right)$ such that

$$
\lim _{n \rightarrow \infty}\left[\mathcal{G}^{V}\left(\left|F_{n}-1\right|^{2}\right)+\mathcal{E}_{\mathcal{A}, V}\left(F_{n}, F_{n}\right)\right]=0 .
$$

To this end, we consider $\rho_{n}:=\sqrt{n^{-1}+\rho^{2}}, n \geq 1$. By (2.3), we have $\rho_{n} \in \mathcal{D}\left(\nabla^{\text {ext }}\right)$ with

$$
\Gamma_{\mathbf{1}}\left(\rho_{n}, \rho_{n}\right)=\frac{\rho^{2}}{\rho_{n}^{2}} \leq 1 .
$$

Let $h \in C_{0}^{\infty}([0, \infty))$ such that $h(r)=1$ for $r \leq 1$ and $h(r)=0$ for $r \geq 2$. We have

$$
F_{n}:=h\left(n^{-1} \log \left[1+\rho_{n}\right]\right) \subset \mathcal{F} C_{0}^{\infty}, \quad n \geq 1 .
$$

It is easy to see that $\mathcal{G}^{V}\left(\left|F_{n}-1\right|^{2}\right) \rightarrow 0$ as $n \rightarrow \infty$ and due to (1.8),

$$
\limsup _{n \rightarrow \infty} \mathcal{E}_{\mathcal{A}, V}\left(F_{n}, F_{n}\right) \leq \limsup _{n \rightarrow \infty} \int_{\mathbb{M}} \frac{\left\|\mathcal{A}_{\eta}\right\|_{L^{2}(\eta)}\left\|h^{\prime}\right\|_{\infty}^{2}}{n^{2}(1+\rho)^{2}} \mathcal{G}^{V}(\mathrm{~d} \eta)=0 .
$$

\section{Proofs of the main results}

In this section, we prove Theorems 2.1, 2.2, 2.5 and Corollary 2.3. 


\subsection{Proof of Theorem 2.1 and a local Poincaré inequality}

Proof of Theorem 2.1. The invalidity of the super Poincaré inequality will be included in the proof of Theorem 2.2(3) for a more general case. So, we only prove (1) and (3).

(a) We first prove $\operatorname{gap}\left(\mathcal{L}_{1,0}\right)=1$, i.e. $\lambda=1$ is the optimal constant for the Poincaré inequality

$$
\mathcal{G}\left(F^{2}\right) \leq \frac{1}{\lambda} \mathcal{E}_{\mathbf{1}, 0}(F, F)+\mathcal{G}(F)^{2}, \quad F \in \mathcal{F} C_{0}^{\infty}
$$

to hold. Let $F(\eta)=f\left(\eta\left(A_{1}\right), \cdots, \eta\left(A_{n}\right)\right)$ for some $f \in C_{0}^{\infty}\left(\mathbb{R}^{n}\right)$ and disjoint $A_{1}, \cdots, A_{n}$. This Poincaré inequality reduces to

$$
\mu^{n}\left(f^{2}\right)-\mu^{n}(f)^{2} \leq \mu^{n}\left(\sum_{i=1}^{n} x_{i}\left|\partial_{i} f\left(x_{1}, \cdots, x_{n}\right)\right|^{2}\right)
$$

where according to (1.2),

$$
\mu^{n}(\mathrm{~d} x):=\prod_{i=1}^{n} \mu_{i}\left(\mathrm{~d} x_{i}\right), \quad \mu_{i}(\mathrm{~d} s)=\gamma_{\nu\left(A_{i}\right)}(\mathrm{d} s):=1_{[0, \infty)}(s) \frac{s^{\nu\left(A_{i}\right)-1} \mathrm{e}^{-s}}{\Gamma\left(\nu\left(A_{i}\right)\right)} \mathrm{d} s, 1 \leq i \leq n .
$$

By the additive property of the Poincaré inequality, it suffices to prove that for every $1 \leq i \leq n, \lambda=1$ is the largest constant satisfying

$$
\mu_{i}\left(f^{2}\right)-\mu_{i}(f)^{2} \leq \frac{1}{\lambda} \int_{0}^{\infty} r f^{\prime}(r)^{2} \mu_{i}(\mathrm{~d} r), \quad f \in C_{0}^{\infty}([0, \infty)) .
$$

This follows from the fact that the generator of the Dirichlet form

$$
\mathcal{E}_{i}(f, g):=\int_{0}^{\infty} r f^{\prime}(r) g^{\prime}(r) \mu_{i}(\mathrm{~d} r), \quad f, g \in W^{1,2}\left([0, \infty), \mu_{i}\right)
$$

is

$$
\mathcal{L}_{i} f(r):=r f^{\prime \prime}(r)+\left(\nu\left(A_{i}\right)-r\right) f^{\prime}(r), \quad r \in[0, \infty),
$$

which has spectral gap 1 with the first eigenfunction $u_{i}(r)=r-\nu\left(A_{i}\right)$.

(b) Let $\operatorname{supp} \nu=\left\{x_{1}, \cdots, x_{n}\right\}$, we have $\delta=\min \left\{\nu\left(\left\{x_{i}\right\}\right): 1 \leq i \leq n\right\}>0$. It suffices to find a universal constant $c_{0}>0$ such that (2.1) holds for

$$
F(\eta):=f\left(\eta\left(\left\{x_{1}\right\}\right), \cdots, \eta\left(\left\{x_{n}\right\}\right)\right), \quad f \in C_{0}^{\infty}\left(\mathbb{R}^{n}\right) .
$$

Letting $\mu^{n}$ and $\mu_{i}$ be as in (4.2) for $A_{i}=\left\{x_{i}\right\}$, (2.1) for this $F$ becomes $\mu^{n}\left(f^{2} \log f^{2}\right) \leq \frac{c_{0}}{1 \wedge \delta} \sum_{i=1}^{n} \int_{[0, \infty)^{n}} s_{i}\left(\partial_{i} f\right)^{2}\left(s_{1}, \cdots, s_{n}\right) \mu^{n}\left(\mathrm{~d} s_{1}, \cdots, \mathrm{d} s_{n}\right)+\mu^{n}\left(f^{2}\right) \log \mu^{n}\left(f^{2}\right)$.

By the additive property of the log-Sobolev inequality, this follows from the following Lemma 4.1 .

Lemma 4.1. For any $a, b>0$, let $\mu_{a}(\mathrm{~d} s):=1_{[0, \infty)}(s) \frac{s^{a-1} \mathrm{e}^{-s}}{\Gamma(a)} \mathrm{d} s$ and $\mu_{a, b}(\mathrm{~d} s):=$ $1_{[0, b]}(s) \frac{\mu_{a}(\mathrm{~d} s)}{\mu_{a}([0, b])}$. Then there exists a constant $c_{0}>0$ such that for any $a, b>0$,

$$
\mu_{a, b}\left(f^{2} \log f^{2}\right) \leq \frac{c_{0}}{a \wedge 1} \int_{0}^{b} s f^{\prime}(s)^{2} \mu_{a, b}(\mathrm{~d} s), \quad f \in C^{1}([0, b]), \mu_{a, b}\left(f^{2}\right)=1 .
$$

Proof. (a) Let $a \geq 2$. We will use the Bakry-Émery criterion on Riemannian manifolds with convex boundary which in particular includes $[0, b]$ for $b>0$. More precisely, let 
$\mathcal{L}_{a} f(s)=s f^{\prime \prime}(s)+(a-s) f^{\prime}(s)$ and $\Gamma_{1}(f, g)(s)=s f^{\prime}(s) g^{\prime}(s)$. By [25, Theorem 1.1(4)] with $\sigma=0$ and $t \rightarrow \infty$, if

$$
\Gamma_{2}(f, f):=\frac{1}{2} \mathcal{L}_{a} \Gamma_{1}(f, f)-\Gamma_{1}\left(\mathcal{L}_{a} f, f\right) \geq K \Gamma_{1}(f, f)
$$

holds for some constant $K>0$ and all $f \in C^{2}([0, b])$, then

$$
\mu_{a, b}\left(f^{2} \log f^{2}\right) \leq \frac{2}{K} \int_{0}^{b} s f^{\prime}(s)^{2} \mu_{a, b}(\mathrm{~d} s), \quad f \in C^{1}([0, b]), \mu_{a, b}\left(f^{2}\right)=1 .
$$

So, the desired inequality (4.3) with $c_{0}=4$ follows since

$$
\begin{aligned}
\Gamma_{2}(f, f)(s) & =s^{2} f^{\prime \prime}(s)^{2}+\frac{a+s}{2} f^{\prime}(s)^{2}+2 s f^{\prime}(s) f^{\prime \prime}(s) \\
& \geq \frac{a+s-2}{2 s} \Gamma_{1}(f, f)(s), \quad s \geq 0
\end{aligned}
$$

so that $\Gamma_{2}(f, f) \geq \frac{1}{2} \Gamma_{1}(f, f)$ when $a \geq 2$.

(b) Let $a \in\left(0, \frac{1}{2}\right]$. By (4.4) we have $\Gamma_{2}(f, f)(s) \geq \frac{a \wedge 2}{4} \Gamma_{1}(f, f)(s)$ for $s \geq 2$. So, by the Bakry-Émery criterion,

$$
\mu_{a, b_{1}}\left(1_{\left[2, b_{1}\right]} f^{2} \log f^{2}\right) \leq \frac{8}{a \wedge 2} \mu_{a, b_{1}}\left(1_{\left[2, b_{1}\right]} \Gamma_{1}(f, f)\right)+\mu_{a, b_{1}}\left(1_{\left[2, b_{1}\right]} f^{2}\right) \log \mu_{a, b_{1}}\left(1_{\left[2, b_{1}\right]} f^{2}\right)
$$

holds for any $b_{1}>2$ and all $f \in C^{1}\left(\left[0, b_{1}\right]\right)$.

On the other hand, for any $b_{2}>0$ and $f \in C^{1}\left(\left[0, b_{2}\right]\right)$ with $\mu_{a, b_{2}}(f)=0$, there exists $r_{0} \in\left[0, b_{2}\right]$ such that $f\left(r_{0}\right)=0$. So, for any $r \in\left[0, b_{2}\right]$ we have

$$
\begin{aligned}
|f(r)| & =\left|\int_{r_{0}}^{r} f^{\prime}(s) \mathrm{d} s\right| \leq\left(\int_{0}^{b_{2}} s f^{\prime}(s)^{2} \mu_{a, b_{2}}(\mathrm{~d} s)\right)^{\frac{1}{2}}\left(\int_{0}^{b_{2}} s^{-a} \mathrm{e}^{s} \Gamma(a) \mathrm{d} s\right)^{\frac{1}{2}} \\
& \leq\left(\frac{\Gamma(a) b_{2}^{1-a} \mathrm{e}^{b_{2}}}{1-a} \mu_{a, b_{2}}\left(\Gamma_{1}(f, f)\right)\right)^{\frac{1}{2}}, \quad r \in\left[0, b_{2}\right] .
\end{aligned}
$$

Therefore, for $\mu_{a, b_{2}}\left(f^{2}\right)=1$ with $\mu_{a, b_{2}}(f)=0$ we have

$$
\begin{aligned}
\mu_{a, b_{2}}\left(f^{2} \log f^{2}\right) & \leq \mu_{a, b_{2}}\left(f^{2}\right) \log \left[\frac{\Gamma(a) b_{2}^{1-a} \mathrm{e}^{b_{2}}}{1-a} \mu_{a, b_{2}}\left(\Gamma_{1}(f, f)\right)\right] \\
& \leq \frac{\Gamma(a) b_{2}^{1-a} \mathrm{e}^{b_{2}}}{1-a} \mu_{a, b_{2}}\left(\Gamma_{1}(f, f)\right)-1 .
\end{aligned}
$$

This implies

$$
\begin{aligned}
& \mu_{a, b_{2}}\left(f^{2} \log f^{2}\right)-\mu_{a, b_{2}}\left(f^{2}\right) \log \mu_{a, b_{2}}\left(f^{2}\right) \\
& \leq \frac{\Gamma(a) b_{2}^{1-a} \mathbf{e}^{b_{2}}}{1-a} \mu_{a, b_{2}}\left(\Gamma_{1}(f, f)\right)-\mu_{a, b_{2}}\left(f^{2}\right), \quad f \in C^{1}\left(\left[0, b_{2}\right]\right), \mu_{a, b_{2}}(f)=0 .
\end{aligned}
$$

In general, for a non-zero function $f \in C^{1}\left(\left[0, b_{2}\right]\right)$, let $\tilde{f}=f-\mu_{a, b_{2}}(f)$. We have (see [2])

$$
\begin{aligned}
& \mu_{a, b_{2}}\left(f^{2} \log f^{2}\right)-\mu_{a, b_{2}}\left(f^{2}\right) \log \mu_{a, b_{2}}\left(f^{2}\right) \\
& \leq \mu_{a, b_{2}}\left(\tilde{f}^{2} \log \tilde{f}^{2}\right)-\mu_{a, b_{2}}\left(\tilde{f}^{2}\right) \log \mu_{a, b_{2}}\left(\tilde{f}^{2}\right)+2 \mu_{a, b_{2}}\left(\tilde{f}^{2}\right) .
\end{aligned}
$$

Combining this with (4.6) and using the Poincaré inequality (4.15) below, we arrive at

$$
\begin{aligned}
& \mu_{a, b_{2}}\left(f^{2} \log f^{2}\right)-\mu_{a, b_{2}}\left(f^{2}\right) \log \mu_{a, b_{2}}\left(f^{2}\right) \\
& \leq\left(\frac{\Gamma(a) b_{2}^{1-a} \mathrm{e}^{b_{2}}}{1-a}+1\right) \mu_{a, b_{2}}\left(\Gamma_{1}(f, f)\right), \quad b_{2}>0, f \in C_{b}^{1}\left(\left[0, b_{2}\right]\right) .
\end{aligned}
$$


In conclusion, when $b \leq 4$, the desired inequality (4.3) for $a \in\left(0, \frac{1}{2}\right]$ follows from (4.8). Finally, for $b \geq 4$ we deduce from (4.5) and (4.8) that for any $f \in C^{1}([0, b])$ with $\mu_{a, b}\left(f^{2}\right)=1$,

$$
\begin{aligned}
& \mu_{a, b}\left(f^{2} \log f^{2}\right)=\frac{\int_{0}^{2} s^{a-1} \mathrm{e}^{-s} \mathrm{~d} s}{\int_{0}^{b} s^{a-1} \mathrm{e}^{-s} \mathrm{~d} s} \mu_{a, 2}\left(f^{2} \log f^{2}\right)+\mu_{a, b}\left(1_{[2, b]} f^{2} \log f^{2}\right) \\
& \leq\left(\frac{\Gamma(a) 2^{1-a} \mathrm{e}^{2}}{1-a}+1\right) \mu_{a, b}\left(1_{[0,2]} \Gamma_{1}(f, f)\right)+\mu_{a, b}\left(1_{[0,2]} f^{2}\right) \log \frac{\Gamma(a)}{\int_{0}^{2} s^{a-1} \mathrm{e}^{-s} \mathrm{~d} s} \\
& \quad+\frac{8}{a \wedge 2} \mu_{a, b}\left(1_{[2, b]} \Gamma_{1}(f, f)\right)+\mu_{a, b}\left(1_{[2, b]} f^{2}\right) \log \frac{\Gamma(a)}{\int_{2}^{b} s^{a-1} \mathrm{e}^{-s} \mathrm{~d} s} \\
& \leq \frac{c_{1}}{a} \int_{0}^{b} s f^{\prime}(s)^{2} \mu_{a, b}(\mathrm{~d} s)+\frac{c_{1}}{a} \mu_{a, b}\left(f^{2}\right),
\end{aligned}
$$

where $c_{1}>0$ is a universal constant independent of $a \in\left(0, \frac{1}{2}\right]$ and $b \geq 4$. Combining this with (4.7) and the Poincaré inequality (4.15) below, we prove the inequality (4.3) for some universal constant $c_{0}>0$ and all $a \in\left(0, \frac{1}{2}\right]$ and $b \geq 4$.

(c) Let $a \in\left(\frac{1}{2}, 2\right)$. In this case, we have $a^{\prime}:=\frac{a}{4} \in\left(0, \frac{1}{2}\right]$, so that by (b) there exists a constant $c_{0}>0$ such that

$$
\mu_{a^{\prime}, b}\left(f^{2} \log f^{2}\right) \leq \frac{c_{0}}{a} \int_{0}^{b} s f^{\prime}(s)^{2} \mu_{a^{\prime}, b}(\mathrm{~d} s), \quad a \in\left(\frac{1}{2}, 2\right), f \in C^{1}([0, b]), \mu_{a^{\prime}, b}\left(f^{2}\right)=1 .
$$

Let $\bar{\mu}_{a^{\prime}, \infty}\left(\mathrm{d} s_{1}, \mathrm{~d} s_{2}, \mathrm{~d} s_{3}, \mathrm{~d} s_{4}\right)=\prod_{i=1}^{4} \mu_{a^{\prime}, \infty}\left(\mathrm{d} s_{i}\right)$, where $\mu_{a^{\prime}, \infty}:=\lim _{b \rightarrow \infty} \mu_{a^{\prime}, b}$ is the Gamma distribution with parameter $a^{\prime}$. By the property of Gamma distributions we have

$\int_{[0, \infty)^{n}} f\left(s_{1}+s_{2}+s_{3}+s_{4}\right) \bar{\mu}_{a^{\prime}, \infty}\left(\mathrm{d} s_{1}, \mathrm{~d} s_{2}, \mathrm{~d} s_{3}, \mathrm{~d} s_{4}\right)=\int_{[0, \infty)} f(s) \mu_{a, \infty}(\mathrm{d} s), \quad f \in \mathcal{B}_{b}([0, \infty))$.

Using (4.9) with $b \rightarrow \infty$ and the additivity property of the log-Sobolev inequality, we obtain

$$
\begin{aligned}
& \bar{\mu}_{a^{\prime}, \infty}\left(F^{2} \log F^{2}\right)-\bar{\mu}_{a^{\prime}, \infty}\left(F^{2}\right) \log \bar{\mu}_{a^{\prime}, \infty}\left(F^{2}\right) \\
& \leq \frac{c_{0}}{a} \int_{0}^{b} \sum_{i=1}^{4} s_{i} \partial_{i} F\left(s_{1}, \cdots, s_{4}\right)^{2} \bar{\mu}_{a^{\prime}, b}\left(\mathrm{~d} s_{1}, \cdots, \mathrm{d} s_{4}\right), \quad F \in C_{b}^{1}\left([0, \infty)^{4}\right) .
\end{aligned}
$$

By an approximation argument we may apply this inequality to

$$
F\left(s_{1}, \cdots, s_{4}\right):=f\left(b \wedge\left(s_{1}+\cdots+s_{4}\right)\right)
$$

for $f \in C^{1}([0, b])$, so that (4.3) is derived.

To prove Theorem 2.2, we consider the local Poincaré inequality for $\mathcal{E}_{\mathbf{1 , 0}}$ on the set $\mathbf{B}_{N}$, by decomposing $\eta$ into the radial part $\eta(E)$ and the simplicial part $\bar{\eta}:=\frac{\eta}{\eta(E)}$. It is well known that under $\mathcal{G}$ these two parts are independent with

$$
\mathcal{G}(\eta(E)<r, \bar{\eta} \in \mathbf{A})=\operatorname{Dir}(\mathbf{A}) \gamma_{\nu(E)}([0, r)), \quad r>0, \mathbf{A} \in \mathcal{B}\left(\mathrm{M}_{1}\right),
$$

where $\gamma_{\nu(E)}(\mathrm{d} s):=1_{[0, \infty)}(s) \frac{s^{\nu(E)-1} \mathrm{e}^{-s}}{\Gamma(\nu(E))} \mathrm{d} s$, and Dir is the Dirichlet measure with intensity measure $\nu$, see for instance [17] for details. According to [16] (see also [17, Proposition 3.3]), we have the Poincaré inequality

$$
\operatorname{Dir}\left(F^{2}\right) \leq \operatorname{Dir}\left(\Gamma^{D}(F, F)\right)+\operatorname{Dir}(F)^{2}, \quad F \in \mathcal{F} C_{0}^{\infty},
$$


where for $F(\eta)=f\left(\eta\left(A_{1}\right), \cdots, \eta\left(A_{n}\right)\right)$ and $\eta \in \mathbb{M}_{1}$,

$$
\Gamma^{D}(F, F)(\eta):=\sum_{i, j=1}^{n}\left[\delta_{i j} \eta\left(A_{i}\right)-\eta\left(A_{i}\right) \eta\left(A_{j}\right)\right] \cdot\left[\left(\partial_{i} f\right)\left(\partial_{j} f\right)\right]\left(\eta\left(A_{1}\right), \cdots, \eta\left(A_{n}\right)\right) .
$$

Lemma 4.2. For any $N>0$,

$$
\mathcal{G}\left(1_{\mathbf{B}_{N}} F^{2}\right) \leq\left(\frac{N^{2}}{4 \nu(E)} \vee 1\right) \mathcal{G}\left(1_{\mathbf{B}_{N}} \Gamma_{\mathbf{1}}(F, F)\right), \quad F \in \mathcal{F} C_{0}^{\infty}, \mathcal{G}\left(1_{\mathbf{B}_{N}} F\right)=0 .
$$

Proof. Since $\mathbf{B}_{N}=\left\{\eta(E) \leq N^{2} / 4\right\}$, (4.10) implies

$$
\int_{\mathbb{M}}\left[1_{\mathbf{B}_{N}} F\right](\eta) \mathcal{G}(\mathrm{d} \eta)=\int_{\mathbb{M}_{1} \times\left[0, N^{2} / 4\right]} F(s \bar{\eta}) \operatorname{Dir}(\mathrm{d} \bar{\eta}) \gamma_{\nu(E)}(\mathrm{d} s), \quad F \in L^{1}\left(1_{\mathbf{B}_{N}} \mathcal{G}\right) .
$$

We observe that (2.1) implies

$$
\gamma_{\nu(E)}\left(1_{[0, r]} f^{2}\right) \leq \int_{0}^{r} s f^{\prime}(s)^{2} \gamma_{\nu(E)}(\mathrm{d} s), \quad r>0, f \in C^{1}([0, r]), \gamma_{\nu(E)}\left(1_{[0, r]} f\right)=0 .
$$

Indeed, applying the Poincaré inequality

$$
\mathcal{G}\left(F^{2}\right) \leq \mathcal{E}_{1,0}(F, F)+\mathcal{G}(F)^{2}
$$

to $F(\eta):=f(\eta(E) \wedge r)$, and noting that for $\tilde{f}(s):=f(s \wedge r)$ we have

$$
\begin{aligned}
& \mathcal{G}\left(F^{i}\right)=\gamma_{\nu(E)}\left(\tilde{f}^{i}\right)=\gamma_{\nu(E)}\left(1_{[0, r]} f^{i}\right)+\gamma_{\nu(E)}((r, \infty)) f(r), i=1,2, \\
& \mathcal{E}_{\mathbf{1}, 0}(F, F)=\int_{0}^{\infty} s \tilde{f}^{\prime}(s)^{2} \mathrm{~d} s=\int_{0}^{N} s f^{\prime}(s)^{2} \mathrm{~d} s,
\end{aligned}
$$

it follows that

$$
\begin{aligned}
& \gamma_{\nu(E)}\left(1_{[0, r]} f^{2}\right)=\gamma\left(\tilde{f}^{2}\right)-\gamma_{\nu(E)}((r, \infty)) f(r)^{2} \\
& \leq \int_{0}^{N} s f^{\prime}(s)^{2} \mathrm{~d} s+\gamma_{\nu(E)}((r, \infty))^{2} f(r)^{2}-\gamma_{\nu(E)}((r, \infty)) f(r)^{2} \leq \int_{0}^{N} s f^{\prime}(s)^{2} \mathrm{~d} s .
\end{aligned}
$$

By the additivity property of the Poincaré inequality, (4.11), (4.14) and (4.15), we obtain that for any $F \in \mathcal{F} C_{0}^{\infty}$ with $\mathcal{G}\left(1_{\mathbf{B}_{N}} F\right)=0$,

$$
\begin{aligned}
\mathcal{G}\left(1_{\mathbf{B}_{N}} F^{2}\right) & \leq \int_{\mathbb{M}_{1} \times\left[0, N^{2} / 4\right]}\left[\frac{1}{\nu(E)} \Gamma^{D}(F(s \cdot), F(s \cdot))(\bar{\eta})+s\left|\frac{\partial}{\partial s} F(s \bar{\eta})\right|^{2}\right] \operatorname{Dir}(\mathrm{d} \bar{\eta}) \gamma_{\nu(E)}(\mathrm{d} s) \\
& =\int_{\mathbf{B}_{N}}\left[\frac{1}{\nu(E)} \Gamma^{D}(F(\eta(E) \cdot), F(\eta(E) \cdot))(\bar{\eta})+\eta(E)\left|\frac{\partial}{\partial \eta(E)} F(\eta(E) \bar{\eta})\right|^{2}\right] \mathcal{G}(\mathrm{d} \eta) .
\end{aligned}
$$

So, it remains to prove

$$
\begin{aligned}
I(\eta) & :=\frac{1}{\nu(E)} \Gamma^{D}(F(\eta(E) \cdot), F(\eta(E) \cdot))(\bar{\eta})+\eta(E)\left|\frac{\partial}{\partial \eta(E)} F(\eta(E) \bar{\eta})\right|^{2} \\
& \leq\left(\frac{N^{2}}{4 \nu(E)} \vee 1\right) \Gamma_{\mathbf{1}}(F, F)(\eta), \quad \eta(E) \leq \frac{N^{2}}{4} .
\end{aligned}
$$

For $F \in \mathcal{F} C_{0}^{\infty}$ with $F(\eta)=f\left(\eta\left(A_{1}\right), \cdots, f\left(A_{n}\right)\right)=f\left(\eta(E) \bar{\eta}\left(A_{1}\right), \cdots, \eta(E) \bar{\eta}\left(A_{n}\right)\right)$, by (4.12) we have

$$
\begin{aligned}
& \Gamma^{D}(F(\eta(E) \cdot), F(\eta(E) \cdot))(\bar{\eta}) \\
& =\sum_{i, j=1}^{n}\left[\delta_{i j} \bar{\eta}\left(A_{i}\right)-\bar{\eta}\left(A_{i}\right) \bar{\eta}\left(A_{j}\right)\right] \eta(E)^{2}\left[\left(\partial_{i} f\right)\left(\partial_{j} f\right)\right]\left(\eta\left(A_{1}\right), \cdots, \eta\left(A_{n}\right)\right) \\
& =\sum_{i, j=1}^{n}\left[\delta_{i j} \eta\left(A_{i}\right) \eta(E)-\eta\left(A_{i}\right) \eta\left(A_{j}\right)\right)\left[\left(\partial_{i} f\right)\left(\partial_{j} f\right)\right]\left(\eta\left(A_{1}\right), \cdots, \eta\left(A_{n}\right)\right) .
\end{aligned}
$$


Moreover,

$$
\begin{aligned}
\eta(E)\left|\frac{\partial}{\partial \eta(E)} F(\eta(E) \bar{\eta})\right|^{2} & =\eta(E)\left|\sum_{i=1}^{n} \bar{\eta}\left(A_{i}\right)\left(\partial_{i} f\right)\left(\eta\left(A_{1}\right), \cdots, \eta\left(A_{n}\right)\right)\right|^{2} \\
& =\frac{1}{\eta(E)} \sum_{i, j=1}^{n} \eta\left(A_{i}\right) \eta\left(A_{j}\right)\left[\left(\partial_{i} f\right)\left(\partial_{j} f\right)\right]\left(\eta\left(A_{1}\right), \cdots, \eta\left(A_{n}\right)\right)
\end{aligned}
$$

So, when $\eta(E) \leq \frac{N^{2}}{4}$ (i.e. $\left.\rho(E) \leq N\right)$,

$$
\begin{aligned}
I(\eta) & \leq \frac{\eta(E) \vee \nu(E)}{\nu(E)}\left(\frac{1}{\eta(E)} \Gamma^{D}(F(\eta(E) \cdot), F(\eta(E) \cdot))(\bar{\eta})+\eta(E)\left|\frac{\partial}{\partial \eta(E)} F(\eta(E) \bar{\eta})\right|^{2}\right) \\
& =\left(1 \vee \frac{N^{2}}{4 \nu(E)}\right) \Gamma_{\mathbf{1}}(F, F)(\eta) .
\end{aligned}
$$

This implies (4.16), and hence finishes the proof.

\subsection{Proofs of Theorem 2.2 and Corollary 2.3}

Proof of Theorem 2.2. We will make a standard split argument by using the local Poincaré inequality (4.13) and the principal eigenvalue of $\mathcal{L}_{\mathcal{A}, V}$ outside $\mathbf{B}_{N}$. To estimate the principal eigenvalue, we recall Hardy's criterion for the first mixed eigenvalue. Consider the following differential operator on $[0, \infty)$ :

$$
\mathcal{L} f(r)=\underline{a}(r) f^{\prime \prime}(r)+\gamma(r) f^{\prime}(r), \quad r \geq 0 .
$$

For any $k>0$ and $n \geq 1$, let $\lambda_{k, n}$ be the first mixed eigenvalue of $\mathcal{L}$ on $[k, k+n]$ with Dirichlet boundary condition at $k$ and Neumann boundary condition at $k+n$. Define

$$
\sigma_{k, n}=\sup _{t \in(k, n+k)} \int_{t}^{n+k} \mathrm{e}^{\int_{k}^{r} \frac{\gamma(s)}{\underline{a}(s)} \mathrm{d} s} \mathrm{~d} r \int_{k}^{t} \frac{1}{\underline{a}(r)} \mathrm{e}^{-\int_{k}^{r} \frac{\gamma(s)}{\underline{a}(s)} \mathrm{d} s} \mathrm{~d} r .
$$

By Hardy's criterion, see for instance [24, Theorem 1.4.2], we have

$$
\frac{1}{\sigma_{k, n}} \geq \lambda_{k, k+n} \geq \frac{1}{4 \sigma_{k, n}}, \quad n \geq 1, k>0 .
$$

Below we prove assertions (1)-(3) respectively.

(1) By (4.13) and a standard perturbation argument, we have

$$
\mathcal{G}^{V}\left(1_{\{\rho \leq N\}} F^{2}\right) \leq \mathcal{G}^{V}\left(1_{\{\rho \leq N\}} F\right)^{2}+\Phi(N) \mathcal{G}^{V}\left(1_{\{\rho \leq N\}} \Gamma_{\mathcal{A}}(F, F)\right), \quad F \in \mathcal{F} C_{0}^{2} .
$$

If $\sigma_{k}<\infty$ for some $k>0$, it suffices to prove the Poincaré inequality

$$
\mathcal{G}^{V}\left(F^{2}\right) \leq C \mathcal{G}^{V}\left(\Gamma_{\mathcal{A}}(F, F)\right), \quad F \in \mathcal{F} C_{0}^{2}, \mathcal{G}^{V}(F)=0
$$

for

$$
C=2 \Phi\left(\psi^{-1}\left(\psi(k)+8 \lambda_{k}^{-1}+1\right)+\lambda_{k}\right)+8 \lambda_{k}^{-1},
$$

where according to (4.17),

$$
\lambda_{k}:=\lim _{n \rightarrow \infty} \lambda_{k, n} \geq \frac{1}{4 \sigma_{k}} .
$$

Let $F \in \mathcal{F} C_{0}^{2}$ such that $\operatorname{supp} F \subset \mathbf{B}_{N_{1}}$ for some constant $N_{1}>k$. For any $N \geq k$, let

$$
F_{N}=F\left[(\psi(\rho)-\psi(N))^{+} \wedge 1\right] .
$$


Then $F_{N}=0$ for $\rho \leq N$ and $F_{N}=F$ for $\psi(\rho) \geq \psi(N)+1$. For $n>N_{1}$, let $u_{n} \geq 0$ be the first mixed eigenfunction of $\mathcal{L}$ on $[k, k+n]$ with Dirichlet boundary condition at $k$ and Neumann boundary condition at $k+n$, such that

$$
u_{n}(k)=u_{n}^{\prime}(k+n)=0, \quad u_{n}^{\prime}(r)>0 \text { for } r \in(k, k+n), \quad \mathcal{L} u_{n}=-\lambda_{k, n} u_{n} \leq 0 .
$$

Combining this with the definition of $\mathcal{L}$ we obtain

$$
\mathcal{L}_{\mathcal{A}, V}\left(u_{n} \circ \rho\right) \geq\left(\mathcal{L} u_{n}\right) \circ \rho, \rho \in[k, k+n] .
$$

So,

$$
\begin{aligned}
& \lambda_{k, n} \mathcal{G}^{V}\left(F_{N}^{2}\right)=-\int_{\{k<\rho<n+k\}} \frac{F_{N}^{2}}{u_{n}^{2} \circ \rho}\left(-\mathcal{L} u_{n}\right) \circ \rho \mathrm{d} \mathcal{G}^{V} \\
& \leq-\int_{\{k<\rho<n+k\}} \frac{F_{N}^{2}}{u_{n} \circ \rho}\left[-\mathcal{L}_{\mathcal{A}, V}\left(u_{n} \circ \rho\right)\right] \mathrm{d} \mathcal{G}^{V} .
\end{aligned}
$$

To apply the integration by parts formula, we approximate $u_{n}$ as follows. Since $u_{n}(k)=$ $u_{n}^{\prime}(k+n)=0$, we may construct a sequence $\left\{u_{n, m}\right\}_{m \geq 1} \subset C^{\infty}([0, \infty))$ such that

$$
\begin{aligned}
& u_{n, m}(r)=u_{n}(r) \text { for } r \in\left[k+m^{-1}, k+n-m^{-1}\right], \\
& u_{n, m}(r)=0 \text { for } r \leq k, u_{n, m}^{\prime}(r)=0 \text { for } r \geq k+n, \\
& \sup _{m \geq 1} \sup _{r \geq k}\left(\left|u_{n, m}^{\prime}(r)\right|+\left|u_{n, m}^{\prime \prime}(r)\right|\right)<\infty .
\end{aligned}
$$

Since $F_{N}=0$ for $\rho \leq N$, (4.21) implies that for any $k<N$,

$$
\begin{aligned}
\lambda_{k, n} \mathcal{G}^{V}\left(F_{N}^{2}\right) & =-\lim _{m \rightarrow \infty} \int_{\{k<\rho<n+k\}} \frac{F_{N}^{2}}{u_{n, m}^{2} \circ \rho}\left(-\mathcal{L} u_{n, m}\right) \circ \rho \mathrm{d} \mathcal{G}^{V} \\
& =\lim _{m \rightarrow \infty} \int_{\mathbb{M}}\left\langle\mathcal{A}_{\eta} \nabla^{e x t} \frac{F_{N}^{2}}{u_{n, m} \circ \rho}(\eta), \nabla^{e x t}\left(u_{n, m} \circ \rho\right)(\eta)\right\rangle_{L^{2}(\eta)} \mathrm{d} \mathcal{G}^{V} .
\end{aligned}
$$

On the other hand, since $\mathcal{A}_{\eta}$ is positive definite due to (H), for any $u \in C^{2}([0, \infty))$ with $u(r)>0$ for $r \geq N$, we have

$$
\begin{aligned}
& \left\langle\mathcal{A}_{\eta} \nabla^{e x t} \frac{F_{N}^{2}}{u \circ \rho}(\eta), \nabla^{e x t}(u \circ \rho)(\eta)\right\rangle_{L^{2}(\eta)} \\
& =\left\langle\mathcal{A}_{\eta} \nabla^{e x t} F_{N}(\eta), \nabla^{e x t} F_{N}(\eta)\right\rangle_{L^{2}(\eta)} \\
& \quad-\left\langle\mathcal{A}_{\eta}\left[\nabla^{e x t} F_{N}-\frac{F_{N}}{u \circ \rho} \nabla^{e x t}(u \circ \rho)\right](\eta), \nabla^{e x t} F_{N}(\eta)-\frac{F_{N}}{u \circ \rho} \nabla^{e x t}(u \circ \rho)(\eta)\right\rangle_{L^{2}(\eta)} \\
& \leq\left\langle\mathcal{A}_{\eta} \nabla^{e x t} F_{N}(\eta), \nabla^{e x t} F_{N}(\eta)\right\rangle_{L^{2}(\eta)} .
\end{aligned}
$$

Combining this with (4.22) and the definition of $F_{N}$, we obtain

$$
\begin{aligned}
& \lambda_{k, n} \mathcal{G}^{V}\left(F_{N}^{2}\right) \leq \int_{\mathbb{M}}\left\langle\mathcal{A}_{\eta} \nabla^{e x t} F_{N}, \nabla^{e x t} F_{N}\right\rangle_{L^{2}(\eta)} \mathrm{d} \mathcal{G}^{V} \\
& \leq 2 \mathcal{E}_{\mathcal{A}, V}(F, F)+2 \int_{\{\psi(N)<\psi(\rho)<\psi(N)+1\}} F^{2} \Gamma_{\mathcal{A}}(\psi(\rho), \psi(\rho)) \mathrm{d} \mathcal{G}^{V} .
\end{aligned}
$$

Multiplying by $\lambda_{k, n}^{-1}$ and letting $n \rightarrow \infty$ leads to

$$
\int_{\mathbb{M}} F_{N}^{2} \mathrm{~d} \mathcal{G}^{V} \leq \frac{2}{\lambda_{k}} \mathcal{E}_{\mathcal{A}, V}(F, F)+\frac{2}{\lambda_{k}} \int_{\{\psi(N)<\psi(\rho)<\psi(N)+1\}} F^{2} \Gamma_{\mathcal{A}}(\psi(\rho), \psi(\rho)) \mathrm{d} \mathcal{G}^{V} .
$$


By the definition of $\psi$ and $\bar{a}$, and noting that $\Gamma_{\mathbf{1}}(\rho, \rho)=1$, we have

$$
\Gamma_{\mathcal{A}}(\psi(\rho), \psi(\rho))(\eta)=\frac{\left\langle\mathcal{A}_{\eta} \nabla^{e x t} \rho(\eta), \nabla^{e x t} \rho(\eta)\right\rangle_{L^{2}(\eta)}}{\bar{a}(\rho(\eta))} \leq \Gamma_{\mathbf{1}}(\rho, \rho)(\eta)=1 .
$$

So, (4.23) implies

$$
\int_{\mathbb{M}} F_{N}^{2} \mathrm{~d} \mathcal{G}^{V} \leq \frac{2}{\lambda_{k}} \mathcal{E}_{\mathcal{A}, V}(F, F)+\frac{2}{\lambda_{k}} \int_{\{\psi(N)<\psi(\rho)<\psi(N)+1\}} F^{2} \mathrm{~d} \mathcal{G}^{V} .
$$

Letting $\lfloor s\rfloor=\sup \{k \in \mathbb{Z}: k \leq s\}$ be the integer part of a real number $s$, we have

$$
\int_{\mathbb{M}} F^{2} \mathrm{~d} \mathcal{G}^{V} \geq \sum_{i=1}^{1+\left\lfloor 8 \lambda_{k}^{-1}\right\rfloor} \int_{\{\psi(k)+i-1<\psi(\rho)<\psi(k)+i]} F^{2} \mathrm{~d} \mathcal{G}^{V} .
$$

Then there exists $N \in\left[k, \psi^{-1}\left(\psi(k)+8 \lambda_{k}^{-1}\right)\right]$ such that

$$
\int_{\{\psi(N)<\psi(\rho)<\psi(N)+1\}} F^{2} \mathrm{~d} \mathcal{G}^{V} \leq \frac{\lambda_{k}}{8} \int_{\mathbb{M}} F^{2} \mathrm{~d} \mathcal{G}^{V},
$$

so that (4.25) yields

$$
\int_{\mathbb{M}} F_{N}^{2} \mathrm{~d} \mathcal{G}^{V} \leq \frac{2}{\lambda_{k}} \mathcal{E}_{\mathcal{A}, V}(F, F)+\frac{1}{4} \int_{\mathbb{M}} F^{2} \mathrm{~d} \mathcal{G}^{V} .
$$

Combining this with (4.18) and noting that $\mathcal{G}^{V}(F)=0$, we may find $N \in\left[k, \psi^{-1}(\psi(k)+\right.$ $\left.\left.8 \lambda_{k}^{-1}\right)\right]$ such that

$$
\begin{aligned}
& \int_{\mathbb{M}} F^{2} \mathrm{~d} \mathcal{G}^{V} \leq \int_{\psi(\rho) \leq \psi(N)+1} F^{2} \mathrm{~d} \mathcal{G}^{V}+\int_{\mathbb{M}} F_{N}^{2} \mathrm{~d} \mathcal{G}^{V} \\
& \leq \Phi\left(\psi^{-1}(\psi(N)+1)\right) \mathcal{E}_{\mathcal{A}, V}(F, F)+\mathcal{G}^{V}\left(1_{\{\psi(\rho) \geq \psi(N)+1\}} F\right)^{2}+\int_{\mathbb{M}} F_{N}^{2} \mathrm{~d} \mathcal{G}^{V} \\
& \leq \Phi\left(\psi^{-1}(\psi(N)+1)\right) \mathcal{E}_{\mathcal{A}, V}(F, F)+2 \int_{\mathbb{M}} F_{N}^{2} \mathrm{~d} \mathcal{G}^{V} \\
& \leq\left(\Phi\left(\psi^{-1}(\psi(N)+1)\right)+\frac{4}{\lambda_{k}}\right) \mathcal{E}_{\mathcal{A}, V}(F, F)+\frac{1}{2} \int_{\mathbb{M}} F^{2} \mathrm{~d} \mathcal{G}^{V} .
\end{aligned}
$$

Since $\Phi(N)$ is increasing in $N \in\left[k, \psi^{-1}\left(\psi(k)+8 \lambda_{k}^{-1}\right)\right]$, this implies (4.19) with

$$
C=2 \Phi\left(\psi^{-1}(\psi(N)+1)\right)+\frac{8}{\lambda_{k}} \leq 2 \Phi\left(\psi^{-1}\left(\psi(k)+8 \lambda_{k}^{-1}+1\right)+\lambda_{k}\right)+8 \lambda_{k}^{-1} .
$$

Then the proof is finished by (4.20).

(2) Assume that $\operatorname{supp} \nu$ is an infinite set. To disprove the super Poincaré inequality, it suffices to construct a sequence $\left\{F_{n}\right\} \subset \mathcal{D}\left(\mathcal{E}_{\mathcal{A}, V}\right)$ such that $\mathcal{G}^{V}\left(F_{n}^{2}\right)>0$ and

$$
C:=\sup _{n \geq 1} \frac{\mathcal{E}_{\mathcal{A}, V}\left(F_{n}, F_{n}\right)}{\mathcal{G}^{V}\left(F_{n}^{2}\right)}<\infty, \lim _{n \rightarrow \infty} \frac{\mathcal{G}^{V}\left(\left|F_{n}\right|\right)^{2}}{\mathcal{G}^{V}\left(F_{n}^{2}\right)}=0 .
$$

Indeed, if (1.11) holds for some $\beta:(0, \infty) \rightarrow(0, \infty)$, then

$$
1 \leq r \frac{\mathcal{E}_{\mathcal{A}, V}\left(F, F_{n}\right)}{\mathcal{G}^{V}\left(F_{n}^{2}\right)}+\beta(r) \frac{\mathcal{G}^{V}\left(\left|F_{n}\right|\right)^{2}}{\mathcal{G}^{V}\left(F_{n}^{2}\right)}, \quad n \geq 1, r>0 .
$$

Combining this with (4.27) and letting $n \rightarrow \infty$, we obtain $1 \leq r C$ for all $r>0$ which is impossible. 
We now show that (4.27) holds for $F_{n}(\eta):=(1-\eta(E))^{+} \frac{\eta\left(A_{n}\right)}{\eta(E)}$, where $\left\{A_{n}\right\}_{n \geq 1}$ are measurable subsets of $E$ such that $\frac{1}{2} \nu(E)>p_{n}:=\nu\left(A_{n}\right) \downarrow 0$ as $n \uparrow \infty$, which exist since $\operatorname{supp} \nu$ is an infinite set.

Obviously, $\left\{F_{n}\right\}_{n \geq 1} \subset \mathcal{D}\left(\mathcal{E}_{\mathcal{A}, V}\right)$. Since $\left\|\mathcal{A}_{\eta}\right\|_{L^{2}(\eta)}+\mathrm{e}^{V(\eta)}+\mathrm{e}^{-V(\eta)}$ is bounded on the set $\{\eta(E) \leq 1\}$, we may find constants $K_{i}, C_{i}>0, i=1,2,3$ such that (4.10) implies for all $n \geq 1$ that

$$
\begin{aligned}
& \mathcal{G}^{V}\left(F_{n}^{2}\right) \geq K_{1} \mathcal{G}\left(F_{n}^{2}\right)=K_{1} \int_{0}^{1} \frac{(1-s)^{2} s^{\nu(E)-1} \mathrm{e}^{-s}}{\Gamma(\nu(E))} \mathrm{d} s \int_{0}^{1} \frac{t^{p_{n}+1}(1-t)^{\nu(E)-p_{n}-1}}{\Gamma\left(p_{n}\right) \Gamma\left(\nu(E)-p_{n}\right)} \mathrm{d} t \geq C_{1} p_{n}, \\
& \mathcal{G}^{V}\left(\left|F_{n}\right|\right)^{2} \leq K_{2} \mathcal{G}\left(\left|F_{n}\right|\right)^{2}=K_{2}\left(\int_{0}^{1} \frac{(1-s) s^{\nu(E)-1} \mathrm{e}^{-s}}{\Gamma(\nu(E))} \mathrm{d} s \int_{0}^{1} \frac{t^{p_{n}}(1-t)^{\nu(E)-p_{n}-1}}{\Gamma\left(p_{n}\right) \Gamma\left(\nu(E)-p_{n}\right)} \mathrm{d} t\right)^{2} \\
& \quad \leq C_{2} p_{n}^{2} \\
& \mathcal{E}_{\mathcal{A}, V}\left(F_{n}, F_{n}\right) \leq K_{3} \mathcal{G}\left(\left\|\nabla^{e x t} F_{n}\right\|_{L^{2}(\eta)}^{2}\right)=K_{3} \int_{\{\eta(E) \leq 1\}} \mathcal{G}(\mathrm{d} \eta) \int_{E}\left|(1-\eta(E))-\eta\left(A_{n}\right)\right|^{2} \mathrm{~d} \eta \\
& \quad \leq 2 K_{3} \int_{\{\eta(E) \leq 1\}}\left[(1-\eta(E))^{2} \eta\left(A_{n}\right)+\eta\left(A_{n}\right)^{2} \eta(E)\right] \mathcal{G}(\mathrm{d} \eta) \\
& \quad \leq 4 K_{3} \int_{\{\eta(E) \leq 1\}} \frac{\eta\left(A_{n}\right)}{\eta(E)} \mathcal{G}(\mathrm{d} \eta) \leq C_{3} p_{n} .
\end{aligned}
$$

Since $p_{n} \downarrow 0$ as $n \uparrow \infty$, we prove (4.27).

(3) The local Poincaré inequality (4.13) implies that for any $F \in \mathcal{F} C_{0}^{\infty}$ with $\mathcal{G}^{V}(F)=0$,

$$
\begin{aligned}
\mathcal{G}^{V}\left(F^{2}\right) & =\mathcal{G}^{V}\left(1_{\mathbf{B}_{N}} F^{2}\right)+\mathcal{G}^{V}\left(F^{2} \cdot 1_{\mathbf{B}_{N}^{c}}\right) \\
& \leq 2 \Phi(N) \mathcal{E}_{\mathcal{A}, V}(F, F)+\frac{1}{\mathcal{G}^{V}\left(\mathbf{B}_{N}\right)} \mathcal{G}^{V}\left(1_{\mathbf{B}_{N}^{c}} F\right)^{2}+\mathcal{G}^{V}(\rho>N)\|F\|_{\infty}^{2} \\
& \leq 2 \Phi(N) \mathcal{E}_{\mathcal{A}, V}(F, F)+\left(\frac{\mathcal{G}^{V}(\rho>N)^{2}}{1-\mathcal{G}^{V}(\rho>N)}+\mathcal{G}^{V}(\rho>N)\right)\|F\|_{\infty}^{2}, \quad N>0 .
\end{aligned}
$$

So, for any $r>0$, taking $N>0$ such that $\frac{\mathcal{G}^{V}(\rho>N)^{2}}{1-\mathcal{G}^{V}(\rho>N)}+\mathcal{G}^{V}(\rho>N) \leq r$, i.e. $\mathcal{G}^{V}(\rho>N) \leq$ $\frac{r}{1+r}$, we prove (1.10).

Proof of Corollary 2.3. Let $r_{0} \in(0, \infty)$ such that $\mathcal{A}_{\eta}=a(\rho(\eta)) \mathbf{1}$ and $V(\eta)=v(\rho(\eta))$ for large $\rho(\eta) \geq r_{0}$. By (2.2) we have

$$
\begin{aligned}
& (\nu-\eta)\left(\mathcal{A}_{\eta} 1\right)=a(\rho(\eta))(\nu(E)-\eta(E))=a(\rho(\eta))\left(\nu(E)-\frac{\rho(\eta)^{2}}{4}\right), \\
& \eta\left(\nabla^{e x t}\left[\mathcal{A}_{\eta} 1(\cdot)\right](\cdot)\right)=a^{\prime}(\rho(\eta)) \eta\left(\frac{1}{\sqrt{\eta(E)}}\right)=\frac{\rho(\eta)}{2} a^{\prime}(\rho(\eta)), \\
& \nabla_{\mathcal{A}_{\eta} 1}^{e x t} V(\eta):=\eta\left(\left[\mathcal{A}_{\eta} 1\right] \nabla^{e x t} V(\eta)\right)=\left(a v^{\prime}\right)(\rho(\eta)) \frac{2 \eta(E)}{\rho(\eta)}=\frac{\rho(\eta)}{2}\left(a v^{\prime}\right)(\rho(\eta)), \\
& \frac{4}{\rho(\eta)^{3}} \eta\left(\mathcal{A}_{\eta} 1\right)=\frac{4 a(\rho(\eta)) \eta(E)}{\rho(\eta)^{3}}=\frac{a(\rho(\eta))}{\rho(\eta)}, \rho(\eta) \geq r_{0} .
\end{aligned}
$$

Then (2.4) implies

$$
\mathcal{L}_{\mathcal{A}_{\eta}, V} \rho(\eta)=\xi(\rho(\eta))
$$

for the given function $\xi$. So, when $\sigma_{k}<\infty$ for some $k>0$, Theorem 2.2(1) implies $\operatorname{gap}\left(\mathcal{L}_{\mathcal{A}, V}\right)>0$.

On the other hand, let $\sigma_{k}=\infty$ for all $k>0$. We have

$$
\lambda_{k}:=\lim _{n \rightarrow \infty} \lambda_{k, n}=0, \quad k>0,
$$


where $\lambda_{k, n}$ is given in the proof of Theorem 2.2. Let $u_{k, n}$ be the corresponding first mixed eigenfunction of $\mathcal{L}$ on $[k, k+n]$ with $u_{k, n}(r)>0$ in $(k, k+n]$, and let

$$
\Theta_{v}(\mathrm{~d} s)=\frac{\mathrm{e}^{v(s)-s} s^{\nu(E)-1}}{\Gamma(\nu(E))} \mathrm{d} s,
$$

such that $\mathcal{L}$ is symmetric in $L^{2}\left([k, k+n], \Theta_{v}\right)$ under the mixed boundary conditions. Then

$$
\int_{k}^{k+n} u_{k, n}(r)^{2} \Theta_{v}(\mathrm{~d} r)=\frac{1}{\lambda_{k, n}} \int_{k}^{k+n} r \underline{a}(r)\left|u_{k, n}^{\prime}(r)\right|^{2} \Theta_{v}(\mathrm{~d} r) .
$$

Letting $F_{k, n}(\eta)=u_{k, n}((\eta(E) \vee k) \wedge(k+n))$, for large enough $k>0$ such that $\mathcal{A}_{\eta}=a(\rho(\eta)) \mathbf{1}$ and $V(\eta)=v(\rho(\eta))$ for $\eta(E) \geq k$, the above formula implies

$$
\mathcal{G}^{V}\left(F_{k, n}^{2}\right)-\mathcal{G}^{V}\left(F_{k, n}\right)^{2} \geq \mathcal{G}^{V}\left(F_{k, n}^{2} \cdot 1_{\{k \leq \rho \leq k+n\}}\right)=\frac{1}{\lambda_{k, n}} \mathcal{E}_{\mathcal{A}, V}\left(F_{n, k}, F_{n, k}\right), \quad n \geq 1 .
$$

Obviously, due to (4.29) this implies $\operatorname{gap}\left(\mathcal{L}_{\mathcal{A}, V}\right)=0$.

\subsection{Proof of Theorem 2.5}

Let $h \in \mathcal{H}$, i.e. $h \in C^{1}([0, \infty))$ with $h(r), h^{\prime}(r)>0$ for $r>0$ such that (2.8) and (2.9) hold. By (2.10) and noting that $V_{h}=V-h(\rho)+c(h)$ where $c(h)$ is a constant such that $\mathcal{G}^{V_{h}}$ is a probability measure, for any $F \in \mathcal{F} C_{0}^{\infty}$ we have

$$
\begin{aligned}
& \mathcal{G}^{V}\left(F^{2} \cdot 1_{\mathbf{B}_{N}}\right)-\frac{\mathcal{G}^{V}\left(F \cdot 1_{\mathbf{B}_{N}}\right)^{2}}{\mathcal{G}^{V}\left(\mathbf{B}_{N}\right)}=\inf _{c \in \mathbb{R},|c| \leq\|F\|_{\infty}} \mathcal{G}^{V}\left(|F-c|^{2} \cdot 1_{\mathbf{B}_{N}}\right) \\
& \leq \mathrm{e}^{h(N)-c(h)} \inf _{c \in \mathbb{R},|c| \leq\|F\|_{\infty}} \mathcal{G}^{V_{h}}\left(|F-c|^{2} \cdot 1_{\mathbf{B}_{N}}\right) \leq \mathrm{e}^{h(N)-c(h)} \inf _{c \in \mathbb{R},|c| \leq\|F\|_{\infty}} \mathcal{G}^{V_{h}}\left(|F-c|^{2}\right) \\
& =\mathrm{e}^{h(N)-c(h)}\left[\mathcal{G}^{V_{h}}\left(F^{2}\right)-\mathcal{G}^{V_{h}}(F)^{2}\right] \leq C(h) \mathrm{e}^{h(N)-c(h)} \mathcal{G}^{V_{h}}\left(\Gamma_{\mathcal{A}}(F, F)\right) \\
& \leq C(h) \mathrm{e}^{h(N)} \mathcal{G}^{V}\left(\Gamma_{\mathcal{A}}(F, F)\right)=C(h) \mathrm{e}^{h(N)} \mathcal{E}_{\mathcal{A}, V}(F, F) .
\end{aligned}
$$

This implies

$$
\mathcal{G}^{V}\left(F^{2} \cdot 1_{\mathbf{B}_{N}}\right) \leq C(h) \mathrm{e}^{h(N)} \mathcal{E}_{\mathcal{A}, V}(F, F)+\frac{\mathcal{G}^{V}\left(F \cdot 1_{\mathbf{B}_{N}}\right)^{2}}{\mathcal{G}^{V}\left(\mathbf{B}_{N}\right)}, \quad F \in \mathcal{D}\left(\mathcal{E}_{\mathcal{A}, V}\right)
$$

Then for any $F \in \mathcal{D}\left(\mathcal{E}_{\mathcal{A}, V}\right)$ with $\mathcal{G}^{V}(F)=0$, we have $\mathcal{G}^{V}\left(F \cdot 1_{\mathbf{B}_{N}}\right)^{2}=\mathcal{G}^{V}\left(F \cdot 1_{\{\rho>N\}}\right)^{2}$ and

$$
\begin{aligned}
& \mathcal{G}^{V}\left(F^{2}\right) \leq \mathcal{G}^{V}\left(F^{2} \cdot 1_{\mathbf{B}_{N}}\right)+\mathcal{G}^{V}\left(F^{2} \cdot 1_{\{\rho>N\}}\right) \\
& \leq C(h) \mathrm{e}^{h(N)} \mathcal{E}_{\mathcal{A}, V}(F, F)+\frac{\mathcal{G}^{V}\left(F \cdot 1_{\mathbf{B}_{N}}\right)^{2}}{\mathcal{G}^{V}\left(\mathbf{B}_{N}\right)}+\mathcal{G}^{V}\left(F^{2} \cdot 1_{\{\rho>N\}}\right) \\
& \leq C(h) \mathrm{e}^{h(N)} \mathcal{E}_{\mathcal{A}, V}(F, F)+\left(\frac{\mathcal{G}^{V}(\rho>N)^{2}}{\mathcal{G}^{V}\left(\mathbf{B}_{N}\right)}+\mathcal{G}^{V}(\rho>N)\right)\|F\|_{\infty}^{2} \\
& \leq C(h) \mathrm{e}^{h(N)} \mathcal{E}_{\mathcal{A}, V}(F, F)+\frac{\mathcal{G}^{V}(\rho>N)}{\mathcal{G}^{V}\left(\mathbf{B}_{N}\right)}\|F\|_{\infty}^{2} .
\end{aligned}
$$

So, for any $r>0$ and $N>0$ such that $\frac{\mathcal{G}^{V}(\rho>N)}{\mathcal{G}^{V}\left(\mathbf{B}_{N}\right)} \leq r$, equivalently $\mathcal{G}^{V}(\rho>N) \leq \frac{r}{1+r}$, we have

$$
\mathcal{G}^{V}\left(F^{2}\right) \leq C(h) \mathrm{e}^{h(N)} \mathcal{E}_{\mathcal{A}, V}(F, F)+r\|F\|_{\infty}^{2}, \quad F \in \mathcal{D}\left(\mathcal{E}_{\mathcal{A}, V}\right), \mathcal{G}^{V}(F)=0, h \in \mathcal{H} .
$$

Therefore, the weak Poincaré inequality (1.10) holds for

$$
\alpha(r):=\inf \left\{C(h) \mathrm{e}^{h(N)}: h \in \mathcal{H}, \mathcal{G}^{V}(\rho>N) \leq \frac{r}{1+r}\right\}, r>0 .
$$




\section{Extensions to the space of finite signed measures}

Consider the space of finite signed measures

$$
\mathbb{M}_{\mathbf{s}}:=\left\{\eta-\eta^{\prime}: \eta, \eta^{\prime} \in \mathbb{M}\right\}
$$

equipped with the topology induced by the map

$$
\eta \mapsto\left(\eta^{+}, \eta^{-}\right) \in \mathbb{M} \times \mathbb{M},
$$

where $\eta^{+}$and $\eta^{-}$are the positive and negative parts of $\eta$ in the Hahn decomposition respectively, and $\mathbb{M} \times \mathbb{M}$ is equipped with the weak topology. So, under this topology $\mathbb{M}_{\mathbf{s}}$ is a Polish space. Note that this topology maybe different from the weak topology, i.e. $\eta_{n} \rightarrow \eta$ if $\eta_{n}(f):=\int_{E} f \mathrm{~d} \eta_{n} \rightarrow \eta(f)$ holds for any $f \in C_{b}(E)$, since the latter on $\mathbb{M}_{\mathbf{s}}$ might be not metrizable, see [19].

To extend the Dirichlet form $\left(\mathcal{E}_{\mathcal{A}, V}, \mathcal{D}\left(\mathcal{E}_{\mathcal{A}, V}\right)\right)$ from $L^{2}\left(\mathcal{G}^{V}\right)$ to $L^{2}\left(\mathcal{G}_{\mathrm{s}}^{V}\right)$ for a probability measure $\mathcal{G}_{\mathbf{s}}^{V}$ with a potential $V$ on $\mathrm{M}_{\mathbf{s}}$, we introduce below the measure $\mathcal{G}_{\mathbf{s}}^{V}$, the extrinsic derivative and the operator $\mathcal{A}$ respectively.

In [18], an analogue to the Lebesgue measure was introduced on $\mathrm{M}_{\mathbf{s}}$ by using the convolution of two weighted Gamma distributions. In the same spirit, we extend the measure $\mathcal{G}$ to $\mathcal{G}_{\mathrm{s}}$ on $\mathrm{M}_{\mathrm{s}}$ as follows:

$$
\int_{\mathbb{M}_{\mathbf{s}}} f(\eta) \mathcal{G}_{\mathbf{s}}(\mathrm{d} \eta)=\int_{\mathbb{M} \times \mathbb{M}} f\left(\eta^{+}-\eta^{-}\right) \mathcal{G}\left(\mathrm{d} \eta^{+}\right) \mathcal{G}\left(\mathrm{d} \eta^{-}\right), \quad f \in \mathcal{B}_{b}\left(\mathbb{M}_{\mathbf{s}}\right) .
$$

Let $\tau(\eta)=\{x \in E: \eta(\{x\}) \neq 0\}$. To ensure that $\tau\left(\eta^{+}\right)$and $\tau\left(\eta^{-}\right)$are disjoint such that $\eta=\eta^{+}-\eta^{-}$is the Hahn decomposition of $\eta$, we will assume that $\nu$ is atomless. In this case, $\tau\left(\eta^{+}\right) \cap \tau\left(\eta^{-}\right)=\emptyset$ for $\mathcal{G} \times \mathcal{G}$-a.e. $\left(\eta^{+}, \eta^{-}\right)$.

Next, we define the extrinsic derivative operator $\left(\nabla^{e x t}, \mathcal{D}\left(\nabla^{e x t}\right)\right)$ as in Definition 1.1 for $\mathbb{M}_{\mathbf{s}}$ replacing $\mathrm{M}$ :

$$
\nabla^{e x t} F(\eta)(x)=\lim _{0 \neq s \rightarrow 0} \frac{F\left(\eta+s \delta_{x}\right)-F(\eta)}{s}, \eta \in \mathbb{M}_{\mathbf{s}} .
$$

Let $\mathcal{F}_{\mathrm{s}} C_{0}^{\infty}$ be the class of cylindrical functions of type

$$
F(\eta):=f\left(\eta^{+}\left(A_{1}\right), \cdots, \eta^{+}\left(A_{n}\right), \eta^{-}\left(A_{1}\right), \cdots, \eta^{-}\left(A_{n}\right)\right), \quad n \geq 1, f \in C_{0}^{\infty}\left(\mathbb{R}^{2 n}\right),
$$

where $\left\{A_{i}\right\}_{1 \leq i \leq n}$ is a measurable partition of $E$, and $\eta=\eta^{+}-\eta^{-}$is the Hahn decomposition. Let

$$
A_{\eta}:=\{x \in E: \eta(\{x\})<0\}, \eta \in \mathbb{M}_{\mathbf{s}} .
$$

It is easy to see that such a function $F$ is extrinsically differentiable with

$$
\nabla^{e x t} F(\eta)=\sum_{i=1}^{2 n}\left(1-2 \cdot 1_{\{i>n\}}\right)\left(\partial_{i} f\right)\left(\eta^{+}\left(A_{1}\right), \cdots, \eta^{+}\left(A_{n}\right), \eta^{-}\left(A_{1}\right), \cdots, \eta^{-}\left(A_{n}\right)\right) 1_{A_{\eta}^{i, n}},
$$

where

$$
A_{\eta}^{i, n}:= \begin{cases}A_{i} \cap A_{\eta}^{c}, & \text { if } i \leq n, \\ A_{i} \cap A_{\eta}, & \text { if } i>n .\end{cases}
$$

Since for any $\eta \in \mathbb{M}_{\mathbf{s}}, A_{\eta+\varepsilon} \delta_{x}=A_{\eta}$ holds for small $\varepsilon>0$ and all $x \in E, \nabla^{\text {ext }} F(\eta)(x)$ is again extrinsically differentiable in $\eta$ with

$$
\begin{aligned}
& \nabla^{e x t}\left[\nabla^{e x t} F(\eta)(x)\right](y)=\sum_{i, j=1}^{2 n}\left[\left(1-2 \cdot 1_{\{i>n\}}\right)\left(1-2 \cdot 1_{\{j>n\}}\right)\right. \\
& \left.\quad \times\left(\partial_{i} \partial_{j} f\right)\left(\eta^{+}\left(A_{1}\right), \cdots, \eta^{+}\left(A_{n}\right), \eta^{-}\left(A_{1}\right), \cdots, \eta^{-}\left(A_{n}\right)\right) 1_{A_{\eta}^{i, n}}(x) 1_{A_{\eta}^{j, n}}(y)\right] .
\end{aligned}
$$


Finally, for any $\eta \in \mathbb{M}_{\mathbf{s}}$, let $\mathcal{A}_{\eta}$ be a positive definite bounded linear operator on $L^{2}(|\eta|)$, where $|\eta|:=\eta^{+}+\eta^{-}$is the total variation of $\eta$. Consider the pre-Dirichlet form

$$
\mathcal{E}_{\mathcal{A}, V}^{\mathbf{s}}(F, G):=\int_{\mathbb{M}_{\mathbf{s}}}\left\langle\mathcal{A}_{\eta} \nabla^{e x t} F(\eta), \nabla^{e x t} G(\eta)\right\rangle_{L^{2}(|\eta|)} \mathrm{d} \mathcal{G}_{\mathbf{s}}^{V}, \quad F, G \in \mathcal{F} C_{0}^{\infty} .
$$

To ensure the closability of this bilinear form, we assume

(H') $\nu$ is atomless, $V \in \mathcal{D}\left(\nabla^{e x t}\right)$ such that $\mathcal{G}_{\mathbf{s}}^{V}$ is a probability measure. Moreover, for any $A \in \mathcal{B}(E)$ and $x \in E, \mathcal{A}_{\eta} 1_{A \cap A_{\eta}^{c}}(x)$ and $\mathcal{A}_{\eta} 1_{A \cap A_{\eta}}(x)$ are extrinsically differentiable in $\eta$ with

$$
\begin{aligned}
\int_{\mathbb{M}_{\mathbf{s}}}[|\eta| & \left(\left|\nabla^{e x t}\left[\mathcal{A}_{\eta} 1_{A \cap A_{\eta}^{c}}\right]\right|+\left|\nabla^{e x t}\left[\mathcal{A}_{\eta} 1_{A \cap A_{\eta}}\right]\right|\right) \\
& \left.+|\eta|\left(\left(\left|\mathcal{A}_{\eta} 1_{A \cap A_{\eta}^{c}}\right|+\left|\mathcal{A}_{\eta} 1_{A \cap A_{\eta}}\right|\right)\left|\nabla^{e x t} V(\eta)\right|\right)\right] \mathcal{G}_{\mathbf{s}}^{V}(\mathrm{~d} \eta)<\infty .
\end{aligned}
$$

Obviously, this assumption is satisfied if $\mathcal{A}_{\eta}=F(\eta) \mathbf{1}$ for some positive bounded extrinsically differentiable function $F$ such that $\mathcal{G}_{\mathbf{s}}^{V}$ is a probability measure with

$$
\int_{\mathbb{M}_{\mathbf{s}}}|\eta|\left(\left|\nabla^{e x t} F(\eta)\right|+\left|\nabla^{e x t} V(\eta)\right|\right) \mathcal{G}_{\mathbf{s}}^{V}(\mathrm{~d} \eta)<\infty .
$$

\subsection{Integration by parts formula}

Theorem 5.1. Assume (H'). Then

$$
\mathcal{E}_{\mathcal{A}, V}^{\mathbf{s}}(F, G)=-\int_{\mathbb{M}_{\mathbf{s}}}\left(G \mathcal{L}_{\mathcal{A}, V}^{\mathbf{s}} F\right) \mathrm{d} \mathcal{G}_{\mathbf{s}}^{V}, \quad F, G \in \mathcal{F}_{\mathbf{s}} C_{0}^{\infty}
$$

holds for

$$
\begin{aligned}
\mathcal{L}_{\mathcal{A}, V}^{\mathbf{s}} F(\eta):= & \int_{E}\left(\left[\nabla^{e x t} V(\eta)(x)\right] \mathcal{A}_{\eta}\left[\nabla^{e x t} F(\eta)\right](x)+\nabla^{e x t}\left(\mathcal{A}_{\eta}\left[\nabla^{e x t} F(\eta)\right](x)\right)(x)\right)|\eta|(\mathrm{d} x) \\
& -\int_{E} \mathcal{A}_{\eta}\left[\nabla^{e x t} F(\eta)\right](x) \eta(\mathrm{d} x) .
\end{aligned}
$$

Consequently, $\left(\mathcal{E}_{\mathcal{A}, V}^{\mathbf{s}}, \mathcal{F}_{\mathbf{s}} C_{0}^{\infty}\right)$ is closable in $L^{2}\left(\mathcal{G}_{\mathbf{s}}^{V}\right)$ and its closure $\left(\mathcal{E}_{\mathcal{A}, V}^{\mathbf{s}}, \mathcal{D}\left(\mathcal{E}_{\mathcal{A}, V}^{\mathbf{s}}\right)\right)$ is a symmetric Dirichlet form with $1 \in \mathcal{D}\left(\mathcal{E}_{\mathcal{A}, V}^{\mathbf{s}}\right)$ and $\mathcal{E}_{\mathcal{A}, V}^{\mathbf{s}}(1,1)=0$.

To prove this result, we introduce the divergence operator associated with $\nabla^{e x t}$.

Definition 5.1. A measurable function $\phi$ on $\mathbb{M}_{\mathbf{s}} \times E$ is said in the domain $\mathcal{D}\left(\operatorname{div}_{\mathbf{s}}^{e x t}\right)$, if for any $x \in E$ we have $\phi(\cdot, x) \in \mathcal{D}\left(\nabla^{e x t}\right)$ and

$$
\int_{\mathbb{M}_{\mathbf{s}}}\left(\int_{E}\left(\left|\nabla^{e x t} \phi(\eta, x)(x)\right|+|\phi(\eta, x)|\right)|\eta|(\mathrm{d} x)\right) \mathcal{G}_{\mathbf{s}}(\mathrm{d} \eta)<\infty .
$$

In this case, the divergence operator is given by

$$
\operatorname{div}_{\mathbf{s}}^{e x t}(\phi)(\eta):=\int_{E} \phi(\eta, x) \eta(\mathrm{d} x)-\int_{E} \nabla^{e x t} \phi(\eta, x)(x)|\eta|(\mathrm{d} x), \quad \eta \in \mathbb{M}_{\mathbf{s}} .
$$

We have the following integration by parts formula for the directional derivative

$$
\nabla_{\phi}^{e x t} F(\eta):=\int_{E}\left[\phi(\eta, x) \nabla^{e x t} F(\eta)(x)\right]|\eta|(\mathrm{d} x), \quad \phi \in \mathcal{D}\left(\operatorname{div}_{\mathbf{s}}^{e x t}\right), F \in \mathcal{D}\left(\nabla^{e x t}\right) .
$$

Lemma 5.2. Let $\phi \in \mathcal{D}\left(\operatorname{div}_{\mathbf{s}}^{e x t}\right)$. Then

$$
\int_{\mathbb{M}_{\mathbf{s}}}\left(\nabla_{\phi}^{e x t} F\right) \mathrm{d} \mathcal{G}_{\mathbf{s}}=\int_{\mathbb{M}_{\mathbf{s}}}\left[F \operatorname{div}_{\mathbf{s}}^{e x t}(\phi)\right] \mathrm{d} \mathcal{G}_{\mathbf{s}}, \quad F \in \mathcal{F}_{\mathbf{s}} C_{0}^{\infty} .
$$


Proof. By a simple approximation argument, we may and do assume that $\phi$ is bounded so that $\left(\mathcal{G}_{\mathrm{s}} \times \nu\right)(|\phi|)<\infty$. For $F \in \mathcal{F}_{\mathrm{s}} C_{0}^{\infty},(5.2)$ implies

$$
\nabla^{e x t} F(\eta)(x)=\nabla^{e x t} F\left(\cdot-\eta^{-}\right)\left(\eta^{+}\right)(x)=-\nabla^{e x t} F\left(\eta^{+}-\cdot\right)\left(\eta^{-}\right)(x), \quad F \in \mathcal{D}\left(\nabla^{e x t}\right) .
$$

Next, for any $\eta^{\prime} \in \mathbb{M}$, let

$$
\phi_{+, \eta^{\prime}}(\eta, x):=\phi\left(\eta^{\prime}-\eta, x\right), \quad \phi_{-, \eta^{\prime}}(\eta, x):=\phi\left(\eta-\eta^{\prime}, x\right), \quad(\eta, x) \in \mathbb{M} \times E .
$$

By (3.6) and (5.11) we obtain

$$
\begin{aligned}
& \operatorname{div}^{e x t}\left(\phi_{-, \eta^{-}}\right)\left(\eta^{+}\right)-\operatorname{div}^{e x t}\left(\phi_{+, \eta^{+}}\right)\left(\eta^{-}\right) \\
& =\int_{E}\left[\phi\left(\eta^{+}-\eta^{-}, x\right)-\nabla^{e x t} \phi\left(\cdot-\eta^{-}, x\right)\left(\eta^{+}\right)(x)\right] \eta^{+}(\mathrm{d} x)-\nu(\phi(\eta, \cdot)) \\
& \quad-\int_{E}\left[\phi\left(\eta^{+}-\eta^{-}, x\right)+\nabla^{e x t} \phi\left(\eta^{+}-\cdot, x\right)\left(\eta^{-}\right)(x)\right] \eta^{-}(\mathrm{d} x)-\nu(\phi(\eta, \cdot)) \\
& =\int_{E} \phi\left(\eta^{+}-\eta^{-}, x\right)\left(\eta^{+}-\eta^{-}\right)(\mathrm{d} x)-\int_{E}\left[\nabla^{e x t}(\cdot, x)\left(\eta^{+}-\eta^{-}\right)(x)\right]\left(\eta^{+}+\eta^{-}\right)(\mathrm{d} x) \\
& =\operatorname{div}_{\mathbf{s}}^{e x t}(\phi)(\eta), \quad \eta=\eta^{+}-\eta^{-} \text {with } \tau\left(\eta^{+}\right) \cap \tau\left(\eta^{-}\right)=\emptyset .
\end{aligned}
$$

Combining this with Lemma 3.3, (5.1) and (5.11), we obtain

$$
\begin{aligned}
& \int_{\mathbb{M}_{\mathbf{s}}}\left(\nabla_{\phi}^{e x t} F\right) \mathrm{d} \mathcal{G}_{\mathbf{s}} \\
& =\int_{\mathbb{M} \times \mathbb{M}} \mathcal{G}\left(\mathrm{d} \eta^{+}\right) \mathcal{G}\left(\mathrm{d} \eta^{-}\right) \int_{E}\left[\phi\left(\eta^{+}-\eta^{-}, x\right) \nabla^{e x t} F\left(\eta^{+}-\eta^{-}\right)(x)\right]\left(\eta^{+}+\eta^{-}\right)(\mathrm{d} x) \\
& =\int_{\mathbb{M}} \mathcal{G}\left(\eta^{-}\right) \int_{\mathbb{M} \times E}\left[\phi\left(\eta^{+}-\eta^{-}, x\right) \nabla^{e x t} F\left(\cdot-\eta^{-}\right)\left(\eta^{+}\right)(x)\right] \eta^{+}(\mathrm{d} x) \\
& \quad-\int_{\mathbb{M}} \mathcal{G}\left(\eta^{+}\right) \int_{\mathbb{M} \times E}\left[\phi\left(\eta^{+}-\eta^{-}, x\right) \nabla^{e x t} F\left(\eta^{+}-\cdot\right)\left(\eta^{-}\right)(x)\right] \eta^{-}(\mathrm{d} x) \\
& =\int_{\mathbb{M} \times \mathbb{M}} F\left(\eta^{+}-\eta^{-}\right)\left[\operatorname{div}^{e x t}\left(\phi_{-, \eta^{-}}\right)\left(\eta^{+}\right)-\operatorname{div}^{e x t}\left(\phi_{+, \eta^{+}}\right)\left(\eta^{-}\right)\right] \mathcal{G}\left(\mathrm{d} \eta^{+}\right) \mathcal{G}\left(\eta^{-}\right) \\
& =\int_{\mathbb{M}_{\mathbf{s}}} F(\eta) \operatorname{div}_{\mathbf{s}}^{e x t}(\phi)(\eta) \mathcal{G}_{\mathbf{s}}(\mathrm{d} \eta) .
\end{aligned}
$$

Proof of Theorem 5.1. Let $F \in \mathcal{F}_{\mathbf{s}} C_{0}^{\infty}$ be given in (5.3), and let

$$
\begin{aligned}
& \phi(\eta, x):=\mathrm{e}^{V(\eta)} \mathcal{A}_{\eta}\left[\nabla^{e x t} F(\eta)\right](x) \\
& =\mathrm{e}^{V(\eta)} \sum_{i=1}^{2 n}\left(1-2 \cdot 1_{\{i>n\}}\right)\left(\partial_{i} f\right)\left(\eta\left(A_{1}, \cdots, \eta\left(A_{n}\right)\right) \mathcal{A}_{\eta} 1_{A_{i}^{\eta}}(x), \quad(\eta, x) \in \mathbb{M}_{\mathbf{s}} \times E .\right.
\end{aligned}
$$

Then (H') and (5.5) imply $\phi \in \mathcal{D}\left(\operatorname{div}_{\mathbf{s}}^{\text {ext }}\right)$. By the definition of $\mathcal{E}_{\mathcal{A}, V}^{\mathbf{s}}$ and Lemma 5.2, for any $G \in \mathcal{F}_{\mathbf{s}} C_{0}^{\infty}$ we have

$$
\begin{aligned}
& \mathcal{E}_{\mathcal{A}, V}^{\mathbf{s}}(F, G)=\int_{\mathbb{M}_{\mathbf{s}}}\left\langle\mathcal{A}_{\eta} \nabla^{e x t} F(\eta), \nabla^{e x t} G(\eta)\right\rangle_{L^{2}(|\eta|)} \mathcal{G}_{\mathbf{s}}^{V}(\mathrm{~d} \eta) \\
& =\int_{\mathbb{M}_{\mathbf{s}}}\left\langle\phi(\eta, \cdot), \nabla^{e x t} G(\eta)\right\rangle_{L^{2}(|\eta|)} \mathcal{G}_{\mathbf{s}}(\mathrm{d} \eta)=\int_{\mathbb{M}_{\mathbf{s}}} G(\eta) \operatorname{div}_{\mathbf{s}}^{e x t}(\phi) \mathcal{G}_{\mathbf{s}}(\mathrm{d} \eta) .
\end{aligned}
$$

This together with (5.10) implies (5.8) for

$$
\begin{aligned}
& \mathcal{L}_{\mathcal{A}, V}^{\mathbf{s}} F(\eta):=-\mathrm{e}^{-V(\eta)} \operatorname{div}_{\mathbf{s}}^{e x t}(\phi)=-\mathrm{e}^{-V(\eta)} \operatorname{div}_{\mathbf{s}}^{e x t}\left(\mathrm{e}^{V(\eta)} \mathcal{A}_{\eta}\left[\nabla^{e x t} F(\eta)\right](\cdot)\right) \\
& =\int_{E}\left(\left[\nabla^{e x t} V(\eta)(x)\right] \mathcal{A}_{\eta}\left[\nabla^{e x t} F(\eta)\right](x)+\nabla^{e x t}\left(\mathcal{A}_{\eta}\left[\nabla^{e x t} F(\eta)\right](x)\right)(x)\right)|\eta|(\mathrm{d} x) \\
& \quad-\int_{E} \mathcal{A}_{\eta}\left[\nabla^{e x t} F(\eta)\right](x) \eta(\mathrm{d} x) .
\end{aligned}
$$


Next, to prove that $1 \in \mathcal{D}\left(\mathcal{E}_{\mathcal{A}, V}^{\mathbf{s}}\right)$ with $\mathcal{E}_{\mathcal{A}, V}^{\mathbf{s}}(1,1)=0$, we take $\left\{f_{n}\right\}_{n \geq 1} \subset C_{0}^{\infty}(\mathbb{R})$ such that $f_{n}(s)=1$ for $|s| \leq n, 0 \leq f_{n} \leq 1$ and $\left\|f_{n}^{\prime}\right\|_{\infty} \leq 1$. Let $F_{n}(\eta):=f_{n}(\eta(E)), n \geq 1$. Then $F_{n} \in \mathcal{F} C_{0}^{\infty}$. By $\left(\mathbf{H}^{\prime}\right)$ we have $\mathcal{G}_{\mathbf{s}}^{V}\left(\left|F_{n}-1\right|^{2}\right) \rightarrow 0$ as $n \rightarrow \infty$, and

$$
\limsup _{n \rightarrow \infty} \mathcal{E}_{\mathcal{A}, V}^{\mathbf{s}}\left(F_{n}, F_{n}\right)=\limsup _{n \rightarrow \infty} \int_{\{|\eta(E)|>n\}}\left\|\mathcal{A}_{\eta} 1\right\|_{L^{1}(|\eta|)} \mathcal{G}_{\mathbf{s}}^{V}(\mathrm{~d} \eta)=0 .
$$

Therefore, $1 \in \mathcal{D}\left(\mathcal{E}_{\mathcal{A}, V}^{2}\right)$ and $\mathcal{E}_{\mathcal{A}, V}^{\mathbf{s}}(1,1)=0$.

\subsection{Functional inequalities for $\mathcal{E}_{1,0}^{\mathbf{s}}$}

For any $N>0$, let $\tilde{\mathbf{B}}_{N}^{\mathbf{s}}=\left\{\eta \in \mathbb{M}_{\mathbf{s}}: \eta^{+}(E) \vee \eta^{-}(E) \leq N\right\}$.

Theorem 5.3. Let $\mathcal{A}=1$ and $V=0$.

(1) $\operatorname{gap}\left(\mathcal{L}_{\mathcal{A}, V}^{\mathbf{s}}\right)=1$, i.e. the following Poincaré inequality

$$
\mathcal{G}_{\mathbf{s}}\left(F^{2}\right) \leq \mathcal{E}_{1,0}^{\mathbf{s}}(F, F)+\mathcal{G}_{\mathbf{s}}(F)^{2}, \quad F \in \mathcal{D}\left(\mathcal{E}_{1,0}^{\mathbf{s}}\right)
$$

holds, and the constant 1 in front of $\mathcal{E}_{1,0}^{\mathbf{s}}(F, F)$ is optimal.

(2) If $\operatorname{supp} \nu$ is infinite, then $\mathcal{E}_{1,0}^{\mathbf{s}}$ does not satisfy the super Poincaré inequality. On the other hand, there exists a constant $c_{0}>0$ such that when $\operatorname{supp} \nu$ is a finite set, the log-Sobolev inequality

$$
\mathcal{G}_{\mathbf{s}}\left(F^{2} \log F^{2}\right) \leq \frac{c_{0}}{1 \wedge \delta} \mathcal{E}_{\mathbf{1}, 0}^{\mathbf{s}}(F, F), \quad F \in \mathcal{D}\left(\mathcal{E}_{\mathbf{1}, 0}\right), \mathcal{G}\left(F^{2}\right)=1
$$

holds, where $\delta:=\min \{\nu(\{x\}): x \in \operatorname{supp} \nu\}$.

(3) For any $N>0$ and $F \in \mathcal{F} C_{0}^{\infty}$ with $\mathcal{G}_{\mathbf{s}}\left(1_{\tilde{\mathbf{B}}_{N}^{\text {s }}} F\right)=0$,

$$
\mathcal{G}_{\mathbf{s}}\left(1_{\tilde{\mathbf{B}}_{N}^{\text {s }}} F^{2}\right) \leq\left(2 \vee \frac{N^{2}}{2 \nu(E)}\right) \mathcal{G}_{\mathbf{s}}\left(1_{\tilde{B}_{N}^{\text {s }}}\left\|\nabla^{e x t} F\right\|_{L^{2}(|\eta|)}^{2}\right) .
$$

Proof. By taking $F(\eta)$ depending only on $\eta^{+}$, it is easy to see that a Poincaré inequality for $\mathcal{E}_{1,0}^{\mathrm{s}}$ implies the same inequality for $\mathcal{E}_{1,0}$. So, the optimality of (5.12), and the invalidity of the super Poincaré inequality when $\operatorname{supp} \nu$ is infinite, follow from Theorem 2.1. It remains to prove the inequalities (5.12), (5.13) and (5.14). According to the additivity property of the Poincaré and log-Sobolev inequalities, these inequalities follow from the corresponding ones of $\mathcal{E}_{1,0}$. For simplicity, below we only prove the first inequality.

Let $\mathcal{F} \in \mathcal{F}_{\mathrm{s}} C_{0}^{\infty}$. By Theorem 2.1, (5.1), (5.7) for $\mathcal{A}=1$ and $V=0$, and using (5.11), we obtain

$$
\begin{aligned}
& \mathcal{G}_{\mathbf{s}}\left(F^{2}\right)=\int_{\mathbb{M}} \mathcal{G}\left(\mathrm{d} \eta^{-}\right) \int_{\mathbb{M}} F\left(\eta^{+}-\eta^{-}\right)^{2} \mathcal{G}\left(\mathrm{d} \eta^{+}\right) \\
& \leq \int_{\mathbb{M} \times \mathbb{M}}\left\|\nabla^{e x t} F\left(\cdot-\eta^{-}\right)\left(\eta^{+}\right)\right\|_{L^{2}\left(\eta^{+}\right)}^{2} \mathcal{G}\left(\mathrm{d} \eta^{+}\right) \mathcal{G}\left(\mathrm{d} \eta^{-}\right)+\int_{\mathbb{M}}\left(\int_{\mathbb{M}} F\left(\eta^{+}-\eta^{-}\right) \mathcal{G}\left(\mathrm{d} \eta^{+}\right)\right)^{2} \mathcal{G}\left(\mathrm{d} \eta^{-}\right) \\
& \leq \int_{\mathbb{M}_{\mathbf{s}}}\left\|\nabla^{e x t} F(\eta)\right\|_{L^{2}\left(\eta^{+}\right)}^{2} \mathcal{G}_{\mathbf{s}}(\mathrm{d} \eta)+\left(\int_{\mathbb{M} \times \mathbb{M}} F\left(\eta^{+}-\eta^{-}\right) \mathcal{G}\left(\mathrm{d} \eta^{+}\right) \mathcal{G}\left(\mathrm{d} \eta^{-}\right)\right)^{2} \\
& \quad+\int_{\mathbb{M}}\left\|\nabla^{e x t}\left[\int_{\mathbb{M}} F\left(\eta^{+}-\cdot\right) \mathcal{G}\left(\mathrm{d} \eta^{+}\right)\right]\left(\eta^{-}\right)\right\|_{L^{2}\left(\eta^{-}\right)}^{2} \mathcal{G}\left(\mathrm{d} \eta^{-}\right) .
\end{aligned}
$$

By the Jensen inequality, we have

$$
\left\|\nabla^{e x t}\left[\int_{\mathbb{M}} F\left(\eta^{+}-\cdot\right) \mathcal{G}\left(\mathrm{d} \eta^{+}\right)\right]\left(\eta^{-}\right)\right\|_{L^{2}\left(\eta^{-}\right)}^{2} \leq \int_{\mathbb{M}}\left\|\nabla^{e x t} F\left(\eta^{+}-\cdot\right)\left(\eta^{-}\right)\right\|_{L^{2}\left(\eta^{-}\right)}^{2} \mathcal{G}\left(\mathrm{d} \eta^{+}\right) .
$$

Therefore,

$$
\mathcal{G}_{\mathbf{s}}\left(F^{2}\right) \leq \mathcal{G}_{\mathbf{s}}(F)^{2}+\int_{\mathbb{M}_{\mathbf{s}}}\left\|\nabla^{e x t} F(\eta)\right\|_{L^{2}\left(\eta^{+}+\eta^{-}\right)}^{2} \mathcal{G}_{\mathbf{s}}(\mathrm{d} \eta)=\mathcal{G}_{\mathbf{s}}(F)^{2}+\mathcal{E}_{\mathbf{1}, 0}^{\mathbf{s}}(F, F) .
$$




\subsection{Functional inequalities for $\mathcal{E}_{\mathcal{A}, V}^{\mathbf{s}}$}

According to the proof of Theorem 2.2 and the local Poincaré inequality (5.14), it seems that we should take

$$
\tilde{\rho}_{\mathbf{s}}(\eta):=2 \sqrt{\eta^{+}(E) \vee \eta^{-}(E)}, \quad \eta \in \mathbb{M}_{\mathbf{s}}
$$

to replace the function $\rho$ on $M$. But by (5.5) we have

$$
\nabla^{e x t} \tilde{\rho}_{\mathbf{s}}(\eta)(x)=\frac{2}{\tilde{\rho}_{\mathbf{s}}(\eta)}\left(1_{\{\eta(E) \geq 0\}} 1_{A_{\eta}^{c}}(x)-1_{\{\eta(E)<0\}} 1_{A_{\eta}}(x)\right),
$$

which is however not extrinsically differentiable in $\eta$, so that $\mathcal{L}_{\mathcal{A}, V} \tilde{\rho}_{\mathbf{s}}$ is not well defined as required. To avoid this problem, below we will use both $\tilde{\rho}_{\mathbf{s}}$ and

$$
\rho_{\mathbf{s}}(\eta):=2 \sqrt{|\eta|(E)}, \quad \eta \in \mathbb{M}_{\mathbf{s}}
$$

which satisfies $\left\|\nabla^{e x t} \rho_{\mathbf{s}}(\eta)\right\|_{L^{2}(|\eta|)}=1$ according to the following lemma.

Lemma 5.4. Let $\rho$ be defined in (5.15) and let $\mathbf{s}(\eta, \cdot):=1-2 \cdot 1_{A_{\eta}}$ for $A_{\eta}$ in (5.4). Then

$$
\nabla^{e x t} \rho_{\mathbf{s}}(\eta)=\frac{2 \mathbf{s}(\eta, \cdot)}{\rho_{\mathbf{s}}(\eta)}, \quad \nabla^{e x t} \mathbf{s}(\eta, x)(y)=0, \quad \eta \in \mathbb{M}_{\mathbf{s}}, x, y \in E .
$$

Consequently, if $\mathcal{A}_{\eta} \mathbf{s}(\eta, \cdot)$ is extrinsically differentiable in $\eta \in \mathbb{M}_{\mathbf{s}}$ with

$$
\sup _{|\eta|(E) \leq r}|\eta|\left(\left|\mathcal{A}_{\eta} \mathbf{s}(\eta, \cdot)\right|+\left|\nabla^{e x t}\left[\mathcal{A}_{\eta} \mathbf{s}(\eta, \cdot)\right](\cdot)\right|\right)<\infty, \quad r \in(0, \infty)
$$

then

$$
\left.\mathcal{L}_{\mathcal{A}, V}^{\mathbf{s}} \rho_{\mathbf{s}}(\eta)=\frac{2}{\rho_{\mathbf{s}}(\eta)}\left[|\eta|\left(\left[\nabla^{e x t} V(\eta)\right] \mathcal{A}_{\eta} \mathbf{s}(\eta, \cdot)\right)+\nabla^{e x t}\left[\mathcal{A}_{\eta} \mathbf{s}(\eta, \cdot)\right](\cdot)\right)-\eta\left(\mathcal{A}_{\eta} \mathbf{s}(\eta, \cdot)\right)\right]
$$

This lemma can be proved by simple calculations using (5.2) and the definition of $\mathcal{L}_{\mathcal{A}, V}$ in Theorem 5.1 , so we omit the details.

By Lemma 5.4, we have

$$
\Gamma_{\mathbf{1}}^{\mathbf{s}}\left(\rho_{\mathbf{s}}, \rho_{\mathbf{s}}\right):=|\eta|\left(\left|\nabla^{e x t} \rho_{\mathbf{s}}\right|^{2}\right)=1, \quad \nabla^{e x t}\left[\nabla^{e x t} \rho_{\mathbf{s}}(\eta)(x)\right](x)=-\frac{4}{\rho_{\mathbf{s}}(\eta)^{3}} .
$$

These coincide with the corresponding properties of $\rho$ on $\mathbb{M}$.

Similarly to (2.5) and (2.6), let

$$
\begin{aligned}
\xi_{\mathbf{s}}(r) & =\inf _{\rho_{\mathbf{s}}(\eta)=r} \mathcal{L}_{\mathcal{A}, V}^{\mathbf{s}} \rho_{\mathbf{s}}(\eta), \quad \underline{a}_{\mathbf{s}}(r)=\inf _{\rho_{\mathbf{s}}(\eta)=r} \inf _{\|\phi\|_{L^{2}(|\eta|)=1}}\left\langle\mathcal{A}_{\eta} \phi, \phi\right\rangle_{L^{2}(|\eta|)}, \\
\bar{a}_{\mathbf{s}}(r) & =\sup _{\rho_{\mathbf{s}}(\eta)=r} \sup _{\|\phi\|_{L^{2}(|\eta|)=1}}\left\langle\mathcal{A}_{\eta} \phi, \phi\right\rangle_{L^{2}(|\eta|)}, \quad r>0 \\
\sigma_{k, \mathbf{s}} & =\sup _{t \geq k} \int_{t}^{\infty} \mathrm{e}^{\int_{k}^{r} \frac{\xi_{\mathbf{s}}(s)}{a_{\mathbf{s}}(s)} \mathrm{d} s} \mathrm{~d} r \int_{k}^{t} \frac{1}{\underline{a}_{\mathbf{s}}(r)} \mathrm{e}^{-\int_{k}^{r} \frac{\xi_{\mathbf{s}}(s)}{\underline{a}_{\mathbf{s}}(s)} \mathrm{d} s} \mathrm{~d} r, \quad k>0
\end{aligned}
$$

Assume that

$$
\psi(t):=\int_{0}^{t}\left[\bar{a}_{\mathbf{s}}(r)\right]^{-\frac{1}{2}} \mathrm{~d} r \uparrow \infty \text { as } t \uparrow \infty .
$$

As in the proof of Theorem 2.2, we may use $\sigma_{k, \mathbf{s}}$ to estimate $\mathcal{G}_{\mathrm{s}}^{V}\left(F_{N}^{2}\right)$ for

$$
F_{N}:=\left[\left(\psi\left(\rho_{\mathbf{s}}\right)-\psi(N)\right)^{+} \wedge 1\right] \cdot F, \quad N>0, F \in \mathcal{F}_{\mathbf{s}} C_{0}^{\infty} .
$$


More precisely, as in (4.20) and (4.26) we conclude that for any $k>0$ there exists $N \in\left[k, \psi^{-1}\left(\psi(k)+32 \sigma_{k, \mathbf{s}}\right)\right]$ such that

$$
\begin{aligned}
\int_{\mathbb{M}_{\mathbf{s}}} F_{N}^{2} \mathrm{~d} \mathcal{G}_{\mathbf{s}}^{V} & \leq \frac{2}{\lambda_{k}} \mathcal{E}_{\mathcal{A}, V}^{\mathbf{s}}(F, F)+\frac{1}{4} \int_{\mathbb{M}} F^{2} \mathrm{~d} \mathcal{G}_{\mathbf{s}}^{V} \\
& \leq 8 \sigma_{k, \mathbf{s}} \mathcal{E}_{\mathcal{A}, V}^{\mathbf{s}}(F, F)+\frac{1}{4} \int_{\mathbb{M}} F^{2} \mathrm{~d} \mathcal{G}_{\mathbf{s}}^{V}
\end{aligned}
$$

On the other hand, we estimate $\mathcal{G}_{\mathbf{s}}^{V}\left(F^{2} \cdot 1_{\left\{\rho_{\mathbf{s}} \leq N\right\}}\right)$ by using the local Poincaré inequality (5.14). Since the bounded set in (5.14) is $\tilde{\mathbf{B}}_{N}^{\mathbf{s}}:=\left\{\tilde{\rho}_{\mathbf{s}} \leq N\right\}$ rather than $\mathbf{B}_{N}^{\mathbf{s}}:=\left\{\rho_{\mathbf{s}} \leq N\right\}$, we change the definition of $\Phi(N)$ into

$$
\Phi_{\mathbf{s}}(N):=\left(2 \vee \frac{N^{2}}{2 \nu(E)}\right) \exp \left[\sup _{\tilde{\rho}_{\mathbf{s}} \leq N} V-\inf _{\tilde{\rho}_{\mathbf{s}} \leq N} V\right] \sup _{\tilde{\rho}_{\mathbf{s}}(\eta) \leq N} \sup _{\|\phi\|_{L^{2}(|\eta|)}=1} \frac{1}{\left\langle\mathcal{A}_{\eta} \phi, \phi\right\rangle_{L^{2}(|\eta|)}}, \quad N>0 .
$$

Noting that $1_{\left\{\rho_{\mathbf{s}} \leq N\right\}} \leq 1_{\left\{\tilde{\rho}_{\mathbf{s}} \leq N\right\}}$, we may apply Theorem 5.3 to bound $\mathcal{G}_{\mathbf{s}}^{V}\left(F^{2} \cdot 1_{\left\{\rho_{\mathbf{s}} \leq N\right\}}\right)$. For instance, corresponding to (4.18) we have

$$
\mathcal{G}_{\mathbf{s}}^{V}\left(F^{2} \cdot 1_{\left\{\rho_{\mathbf{s}} \leq N\right\}}\right) \leq \mathcal{G}_{\mathbf{s}}^{V}\left(F^{2} \cdot 1_{\left\{\tilde{\rho}_{\mathbf{s}} \leq N\right\}}\right) \leq \mathcal{G}_{\mathbf{s}}^{V}\left(1_{\left\{\tilde{\rho}_{\mathbf{s}} \leq N\right\}} F\right)^{2}+\Phi_{\mathbf{s}}(N) \mathcal{E}_{\mathcal{A}, V}^{\mathbf{s}}(F, F) .
$$

Combining this with (5.20) we may extend assertions of Theorem 2.2 to the present setting as follows, where when $\operatorname{supp} \nu$ is infinite the super Poincaré can be disproved as in the proof of Theorem 2.2(2) by taking $F_{n}(\eta)=\left(1-\eta^{+}(E)\right)^{+} \frac{\eta^{+}\left(A_{n}\right)}{\eta^{+}(E)}$ for $0<\nu\left(A_{n}\right) \downarrow 0$. Moreover, one may also extend Corollaries 2.3-2.4 and Theorem 2.5. We omit the details to save space.

Theorem 5.5. In addition to $\left(\mathbf{H}^{\prime}\right)$, assume that $\mathcal{A}_{\eta} \mathbf{s}(\eta, \cdot)$ is extrinsically differentiable in $\eta$ such that (5.16) holds. Moreover, assume that $\underline{a}_{\mathbf{s}}$ and $\bar{a}_{\mathbf{s}}$ in (5.18) are such that $\underline{a}_{\mathbf{s}}^{-1}(r)$ is locally bounded in $r \geq 0$ and (5.19) holds.

(1) If $\lim _{k \rightarrow \infty} \sigma_{k, \mathbf{s}}<\infty$, then

$$
\operatorname{gap}\left(\mathcal{L}_{\mathcal{A}, V}^{\mathbf{s}}\right) \geq \sup \left\{\frac{1}{2 \Phi_{\mathbf{s}}\left(\psi^{-1}\left(\psi(k)+32 \sigma_{k, \mathbf{s}}+1\right)\right)+32 \sigma_{k, \mathbf{s}}}: k>0\right\}>0 .
$$

(2) If $\operatorname{supp} \nu$ contains infinitely many points, then $\mathcal{E}_{\mathcal{A}, V}^{\mathbf{s}}$ does not satisfy the super Poincaré inequality.

(3) The weak Poincaré inequality (1.10) holds for $\left(\mathcal{E}_{\mathcal{A}, V}^{\mathbf{s}}, \mathcal{G}_{\mathbf{s}}^{V}\right)$ replacing $\left(\mathcal{E}_{\mathcal{A}, V}, \mathcal{G}^{V}\right)$ and

$$
\alpha(r):=\inf \left\{2 \Phi_{\mathbf{s}}(N): \mathcal{G}_{\mathbf{s}}^{V}(\rho>N) \leq \frac{r}{1+r}\right\}, r>0
$$

\section{References}

[1] S. Albeverio, Y. G. Kondratiev, M. Röckner, Analysis and geometry on configuration spaces, J. Funct. Anal. 154 (1998) 444-500. MR-1612725

[2] D. Bakry, L'hypercontractivité et son utilisation en théorie des semigroupes, École d'été de Probabilités de Saint Flour XXII. Lecture Notes in Math. 1581, 1-114. Springer, Berlin 1994. MR-1307413

[3] D. Bakry, M. Émery, Hypercontractivité de semi-groupes de diffusion, C. R. Acad. Sci. Paris. Sér. I Math. 299 (1984), 775-778. MR-0772092

[4] M. Fukushima, Y. Oshima, M. Takeda, Dirichlet Forms and Symmetric Markov Processes, de Gruyter, 2011. MR-2778606

[5] I. M. Gelfand, M. I. Graev, A. M. Vershik, Models of representations of current groups, in “Representations of Lie Groups and Lie Algebras" (A. A. Kirillov, Ed.), pp. 121-179, Akadémiai Kiadó, Budapest, 1985. MR-0829048 
[6] L. Gross, Logarithmic Sobolev inequalities and contractivity properties of semigroups, in “Dirichlet Forms", Lecture Notes in Math. 1563 (Springer, Berlin), pp. 54-88. MR-1292277

[7] D. Hagedorn, Y. Kondratiev, E. Lytvynov, A. Vershik, Laplace operators in gamma analysis, in "Stochastic and Infinite Dimensional Analysis" (C. C. Bernido et al. Eds.), pp. 119-147, Birkhäuser, 2016. MR-3708379

[8] D. Hagedorn, Y. Kondratiev, T. Pasurek, M. Röckner, Gibbs states over the cone of discrete measures, J. Funct. Anal. 264 (2013), 2550-2583. MR-3041709

[9] Y. Kondratiev, E. Lytvynov, A. Vershik, Laplace operators on the cone of Radon measures, J. Funct. Anal. 269 (2015), 2947-2976. MR-3394626

[10] J. Mecke, Stationäre zufällige Maße auf lokalkompakten Abelschen Gruppen, Z. Wahrsch. verw. Geb. 9 (1967), 36-58. MR-0228027

[11] L. Overbeck, M. Röckner, B. Schmuland, An analytic approach to Fleming-Viot processes with interactive selection, Ann. Probab. 23 (1995), 1-36. MR-1330758

[12] P. Ren, F.-Y. Wang, Spectral gap for measure-valued diffusion processes, J. Math. Anal. Appl. 485 (2020). MR-4024966

[13] M. Röckner, F.-Y. Wang, Weak Poincaré inequalities and convergence rates of Markov semigroups, J. Funct. Anal. 185 (2001), 564-603. MR-1856277

[14] M. Röckner, F.-Y. Wang, Spectrum for a class of (nonsymmetric) diffusion operators, Bull. London Math. Soc. 36 (2004), 95-104. MR-2011983

[15] O. S. Rothaus, Logarithmic Sobolev inequalities and the spectrum of Schrödinger operators, J. Funct. Anal. 42 (1981), 110-120. MR-0620582

[16] N. Shimakura, Équations différentielles provenant de la génétique des populations, Tôhoku Math. J. 29 (1977), 287-318. MR-0504058

[17] W. Stannat, On validity of the log-Sobolev inequality for symmetric Fleming-Viot operators, Ann. Probab. 28 (2000), 667-684. MR-1782270

[18] N. V. Tsilevich, A. M. Vershik, M. Yor, An infinite-dimensional analogue of the Lebesgue measure and distinguished properties of the gamma process, J. Funct. Anal. 185 (2001), 274-296. MR-1853759

[19] V. S. Varadarajan, Weak convergence of measures on separable metric spaces, Sankhyā 19 (1958), 15-22. MR-0094838

[20] A. M. Vershik, I. M. Gelfand, M. I. Graev, A commutative model of the representation of the group of currents $S L(2, \mathbb{R})^{X}$ connected with a unipotent subgroup, Funct. Anal. Appl. 17 (1983), 137-139. MR-0705049

[21] F.-Y. Wang, Functional inequalities for empty essential spectrum, J. Funct. Anal. 170 (2000), 219-245. MR-1736202

[22] F.-Y. Wang, Functional inequalities, semigroup properties and spectrum estimates, Inf. Dim. Anal. Quant. Probab. Relat. Top. 3 (2000), 263-295. MR-1812701

[23] F.-Y. Wang, Functional inequalities on abstract Hilbert spaces and applications, Math. Zeit. 246 (2004), 359-371. MR-2031460

[24] F.-Y. Wang, Functional Inequalities, Markov Semigroups and Spectral Theory, Science Press, Beijing (2005).

[25] F.-Y. Wang, Semigroup properties for the second fundamental form, Doc. Math. 15 (2010), 543-559. MR-2679065

[26] F.-Y. Wang, W. Zhang, Nash inequality for Diffusion processes associated with Dirichlet distributions, Front. Math. China 14 (2019), 1317-1338. MR-4048048

Acknowledgments. The author would like to thank Professor Shui Feng for valuable conversations, as well as the referee for very careful reading, helpful comments and corrections. 


\section{Electronic Journal of Probability Electronic Communications in Probability}

\section{Advantages of publishing in EJP-ECP}

- Very high standards

- Free for authors, free for readers

- Quick publication (no backlog)

- Secure publication $\left(\mathrm{LOCKSS}^{1}\right)$

- Easy interface (EJMS²)

\section{Economical model of EJP-ECP}

- Non profit, sponsored by $\mathrm{IMS}^{3}, \mathrm{BS}^{4}$, ProjectEuclid ${ }^{5}$

- Purely electronic

\section{Help keep the journal free and vigorous}

- Donate to the IMS open access fund ${ }^{6}$ (click here to donate!)

- Submit your best articles to EJP-ECP

- Choose EJP-ECP over for-profit journals

\footnotetext{
${ }^{1}$ LOCKSS: Lots of Copies Keep Stuff Safe http://www. lockss.org/

${ }^{2}$ EJMS: Electronic Journal Management System http://www.vtex.lt/en/ejms.html

${ }^{3}$ IMS: Institute of Mathematical Statistics http://www.imstat.org/

${ }^{4}$ BS: Bernoulli Society http://www. bernoulli-society.org/

${ }^{5}$ Project Euclid: https://projecteuclid.org/

${ }^{6}$ IMS Open Access Fund: http://www.imstat.org/publications/open.htm
} 